\title{
JACOBI-DAVIDSON TYPE METHODS FOR GENERALIZED EIGENPROBLEMS AND POLYNOMIAL EIGENPROBLEMS *
}

\author{
GERARD L. G. SLEIJPEN ${ }^{1}$, ALBERT G. L. BOOTEN ${ }^{2}$, \\ DIEDERIK R. FOKKEMA ${ }^{1,3}$, and HENK A. VAN DER VORST ${ }^{1}$ \\ ${ }^{1}$ Mathematical Institute, Utrecht University, P.O. Box 80.010, NL-3508 TA Utrecht, The \\ Netherlands.email: sleijpen@math.ruu.nl, vorst@math.ruu.nl \\ ${ }^{2}$ CWI, P.O. Box 94079, NL-1090 GB Amsterdam, The Netherlands \\ ${ }^{3}$ Present address: ISE Integrated Systems Engineering AG, Technopark Zürich, \\ Technoparkstrasse 1, CH-8005 Zürich, Switzerland. email: fokkema@ise.ch
}

\begin{abstract}
.
In this paper we will show how the Jacobi-Davidson iterative method can be used to solve generalized eigenproblems. Similar ideas as for the standard eigenproblem are used, but the projections, that are required to reduce the given problem to a small manageable size, need more attention. We show that by proper choices for the projection operators quadratic convergence can be achieved. The advantage of our approach is that none of the involved operators needs to be inverted. It turns out that similar projections can be used for the iterative approximation of selected eigenvalues and eigenvectors of polynomial eigenvalue equations. This approach has already been used with great success for the solution of quadratic eigenproblems associated with acoustic problems.
\end{abstract}

AMS subject classification: $65 \mathrm{~F} 15,65 \mathrm{~N} 25$.

Key words: Eigenvalues and eigenvectors, eigenproblem, generalized eigenproblem, quadratic eigenproblem, polynomial eigenproblem, Jacobi-Davidson method, Ritz values, harmonic Ritz values.

Our friend Albert died on November 12, 1995

\section{Introduction.}

The Jacobi-Davidson method [29] constructs iteratively approximations of certain eigenvectors. It finds the approximate eigenvector as "best" approximations in some search subspace.

In this paper we are interested in numerical methods to compute accurate approximations of the solutions $(\mu, \lambda, \mathbf{x})$ of the following generalized eigenvalue problem

$$
\mu \mathbf{A x}=\lambda \mathbf{B x},
$$

${ }^{*}$ Received November 1995. Revised March 1996. 
where $\mathbf{A}$ and $\mathbf{B}$ are $n \times n$ matrices, $\mathbf{x}$ is a non-trivial $n$-vector and $(\mu, \lambda)$ is an element in the one dimensional complex projective plane (one may think of $\mu$ and $\lambda$ in the complex plane, scaled such that $\mu \in[0,1]$ and $|\mu|^{2}+|\lambda|^{2}=1$ ). Typically, $n$ is large and $\mathbf{A}$ and $\mathbf{B}$ are sparse.

For the moment, for ease of presentation, we assume $\mathbf{B}$ to be non-singular and we scale $\mu$ to be 1 and take $\lambda \in \mathbb{C}$.

General remarks. Observe that both the eigenproblem $\mathbf{A x}=\lambda \mathbf{x}$, as well as the inverse eigenproblem $(1 / \lambda) \mathbf{x}=\mathbf{A}^{-1} \mathbf{x}$ (assuming that $\mathbf{A}$ is non-singular) fit in the frame of the generalized eigenproblem.

Conversely, for non-singular $\mathbf{B}$, the generalized eigenproblem can be formulated as a eigenproblem, $\mathbf{B}^{-1} \mathbf{A x}=\lambda \mathbf{x}$ or $\mathbf{A B}^{-1} \mathbf{w}=\lambda \mathbf{w}$ with $\mathbf{x}=\mathbf{B w}$.

If $\mathbf{B}$ is positive definite (implying $\mathbf{B}$ is hermitian), then, in order to maintain a possible symmetry of the matrix $\mathbf{A}$, one might wish to represent the generalized eigenproblem as an eigenproblem of $\mathbf{B}^{-\frac{1}{2}} \mathbf{A B}^{-\frac{1}{2}}: \mathbf{B}^{-\frac{1}{2}} \mathbf{A} \mathbf{B}^{-\frac{1}{2}} \mathbf{w}=\lambda \mathbf{w}$ with $\mathbf{x}=\mathbf{B}^{-\frac{1}{2}} \mathbf{w}$.

Since the generalized eigenproblem is "symmetric" in A and B, similar observations can be made, interchanging $\mathbf{A}$ and $\mathbf{B}$, in case $\mathbf{A}$ is non-singular or positive definite.

\section{The updating process for the approximate eigenvector.}

The idea of the Jacobi-Davidson method in [29] is to construct a correction, for a given eigenvector approximation, in a subspace orthogonal to the given approximation. The computation of the correction in a given subspace is done in a Davidson manner; the idea to look for orthogonal corrections is Jacobi's, hence the name for the method.

Suppose we have a non-zero Ritz approximation $\mathbf{u}$ of an eigenvector $\mathbf{x}$ with Ritz value $\vartheta$ corresponding to the eigenvalue $\lambda$ associated to $\mathbf{x}$. Then from the Ritz Galerkin conditions it follows that

$$
\mathbf{r} \equiv \mathbf{A} \mathbf{u}-\vartheta \mathbf{B u} \perp \mathbf{u}
$$

Then the goal is to find an update $\mathbf{z}$ for $\mathbf{u}$ in the space orthogonal to $\mathbf{u}$, such that

$$
\mathbf{z} \perp \mathbf{u} \text { for which } \mathbf{A}(\mathbf{u}+\mathbf{z})=\lambda \mathbf{B}(\mathbf{u}+\mathbf{z}),
$$

and $\mathbf{x}$ is a scalar multiple of $\mathbf{u}+\mathbf{z}$. By taking the inner product of the equation in $(2.2)$ with the given $\mathrm{u}$, and by projecting the same equation onto the space orthogonal to $\mathbf{u}$, with the projector $\mathbf{I}-\frac{\mathbf{u u}^{*}}{\mathbf{u}^{*} \mathbf{u}}$, we obtain two coupled equations for $\lambda$ and $\mathbf{z}$ :

$$
\left\{\begin{array}{c}
\lambda=\frac{\mathbf{u}^{*} \mathbf{A}(\mathbf{u}+\mathbf{z})}{\mathbf{u}^{*} \mathbf{B}(\mathbf{u}+\mathbf{z})} \\
\text { (b) } \mathbf{z} \perp \mathbf{u} \text { and }\left.\left(\mathbf{I}-\frac{\mathbf{u} \mathbf{u}^{*}}{\mathbf{u}^{*} \mathbf{u}}\right)(\mathbf{A}-\lambda \mathbf{B})\right|_{\mathbf{u}^{\perp}} \mathbf{z}=-(\mathbf{a}-\lambda \mathbf{b}),
\end{array}\right.
$$

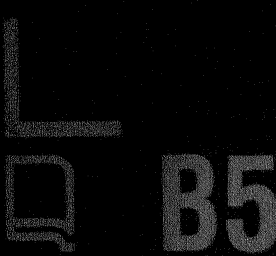


where $\left.\right|_{u+}$ indicates that the action of the matrix is restricted to vectors orthogonal to $\mathbf{u}$, and the vectors $\mathbf{a}$ and $\mathbf{b}$ are defined $b y$

$$
\alpha \equiv \frac{\mathbf{u}^{*} \mathbf{A u}}{\mathbf{u}^{*} \mathbf{u}}, \quad \mathbf{a} \equiv \mathbf{A u}-\alpha \mathbf{u}, \quad \beta=\frac{\mathbf{u}^{*} \mathbf{B u}}{\mathbf{u}^{*} \mathbf{u}}, \quad \mathbf{b} \equiv \mathbf{B u}-\beta \mathbf{u} .
$$

Note that $\alpha, \beta, \mathrm{a}$ and $\mathrm{b}$ can be readily computed. Moreover, because of the Ritz-Galerkin condition 2.1 we have

$$
\vartheta=\frac{\alpha}{\beta} \text { and } \quad \mathbf{r}=\mathbf{a}-\vartheta \mathrm{b} \text {. }
$$

Following another suggestion of Jacobi [15], made for the standard eigenproblem for diagonally dominant symmetric matrices, it was proposed in [29], for general matrices, to solve this system iteratively, computing $\lambda_{j}((2.3 . a))$ with $\mathbf{z}$ replaced by $\mathbf{z}_{j}$, and computing $\mathbf{z}_{j+1}$ from (2.3.b) with $\lambda$ replaced by $\lambda_{j}$ (the Jacobi orthogonal correction method).

With $\mathbf{z}_{0}=0$ as an initial guess we have that $\lambda_{0}=\vartheta$ and that $\mathbf{z}_{1}$ is the solution of the following correction equation.

$$
\mathbf{z}_{1} \perp \mathbf{u} \text { and }\left(\mathbf{I}-\frac{\mathbf{u} \mathbf{u}^{*}}{\mathbf{u}^{*} \mathbf{u}}\right)(\mathbf{A}-\vartheta \mathbf{B})_{\mathbf{u}^{\perp}} \mathbf{z}_{1}=-\mathbf{r} .
$$

Since $\mathbf{z}_{j}$ is only an approximation for the exact correction $\mathbf{z}$ there is no need to solve (2.3.b) (nor the one in (2.4)) very accurately: for instance, we may use a few steps of an iterative method to solve linear systems of equations (e.g., GMRES [25] or Gauss-Jacobi [14], as Jacobi did for the symmetric standard eigenproblem [15]).

In the computation of this approximate $\mathbf{z}_{j}$, one may use the fact that

$$
\left(\mathbf{I}-\frac{\mathbf{u} \mathbf{u}^{*}}{\mathbf{u}^{*} \mathbf{u}}\right) \mathbf{z} \perp \mathbf{u} \text { for any } \mathbf{z},
$$

for the construction of an approximation $\mathbf{z}_{j}$ that is orthogonal to $\mathbf{u}$. The expression in (2.3.a) offers the possibility to update the approximation of the eigenvalue during the phase of solving approximately the linear system in (2.3.b).

In a related context, Davidson [7] pointed out (see also [29]) that the speed of convergence may be improved if we do not restrict ourselves to simply finding the new approximation by correcting $\mathbf{u}^{(k)}=\mathbf{u}$ with an approximate correction $\mathbf{z}_{j}$, but instead compute the Ritz approximations with respect to the subspace spanned by all previous corrections. For computational reasons (efficiency, stability, ...), it is more convenient to use an orthogonal basis $\mathbf{v}_{1}, \ldots, \mathbf{v}_{k+1}$ of vectors that span the same subspace as the set of approximating eigenvectors. This leads to the Jacobi-Davidson strategy, that will be further explained in $\$ 4$.

If we update $\mathbf{u}$ in each step by the exact solution $\mathbf{z}_{1}$ of the correction equation (2.4) (that is, with $\mathbf{u}_{k}=\mathbf{u}$, in the next step we take $\mathbf{u}=\mathbf{u}_{k+1}=\mathbf{u}_{k}+\mathbf{z}_{1}$ ), then we obtain an asymptotically quadratic convergent process, when $\mathbf{B}=\mathbf{I}$ (see Theorem 3.2 , or $[29, \S 4.1])$. To retain quadratic convergence also for the case where $\mathbf{B} \neq \mathbf{I}$, we have to adjust the projection $\mathbf{I}-\frac{\mathbf{u} \mathbf{u}^{*}}{\mathbf{u}^{*} \mathbf{u}}$. We will address the choice of the proper projection in the next section.

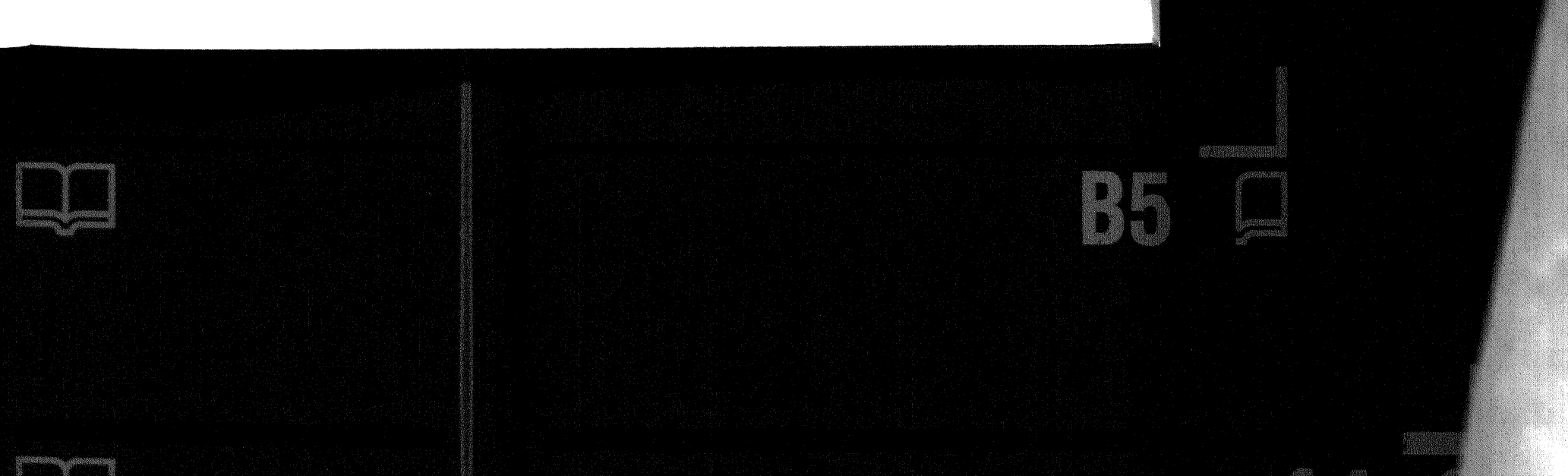




\section{Other projections for the eigenvector approximations.}

Suppose we have a non-trivial approximation $\mathbf{u}$ of an eigenvector $\mathbf{x}$ with approximation $\vartheta$ of the eigenvalue $\lambda$ associated to $\mathbf{x}$. Assume also that we have a vector $\mathrm{w}$ for which

$$
\mathbf{r} \equiv \mathbf{A u}-\vartheta \mathbf{B u} \perp \mathbf{w}
$$

We look for an update $\mathbf{z}$ of $\mathbf{u}$ in the space orthogonal to some $\tilde{\mathbf{u}}(\widetilde{\mathbf{u}} \not \mathcal{u})$ : we are interested in

$$
\mathbf{z} \perp \tilde{\mathbf{u}} \text { for which } \mathbf{A}(\mathbf{u}+\mathbf{z})=\lambda \mathbf{B}(\mathbf{u}+\mathbf{z}) .
$$

As in $\S 2$ we have, except for a scalar multiple, $\mathbf{x}=\mathbf{u}+\mathbf{z}$. The introduction of $\tilde{\mathbf{u}}$ is not so obvious at the moment, we could simply have taken the vector $\mathbf{u}$. It will appear that other choices for $\widetilde{\mathbf{u}}$ may be convenient, like $\widetilde{\mathbf{u}}=\mathrm{Bu}$.

For similar reasons we select a $\tilde{\mathbf{w}} \not \subset \mathbf{w}$, and we consider the projections

$$
\mathbf{P} \equiv \frac{\widetilde{\mathbf{w}} \mathbf{w}^{*}}{\mathbf{w}^{*} \widetilde{\mathbf{w}}} \quad \text { and } \quad \mathbf{Q} \equiv \frac{\mathbf{u} \widetilde{\mathbf{u}}^{*}}{\widetilde{\mathbf{u}}^{*} \mathbf{u}}
$$

The projection $\mathbf{Q}$ splits the space $\mathbb{C}^{n}$ into the subspace spanned by $\mathbf{u}$ and the orthogonal complement of $\tilde{\mathbf{u}}$, that is, $\mathbf{v}=\mathbf{Q v}+(\mathbf{I}-\mathbf{Q}) \mathbf{v}, \mathbf{Q v}=\gamma \mathbf{u}$ and $(\mathbf{I}-\mathbf{Q}) \mathbf{v} \perp \tilde{\mathbf{u}}$ for each $\mathbf{v}$. Similarly, the projection $\mathbf{P}$ splits the space into the subspace spanned by $\tilde{\mathbf{w}}$ and the orthogonal complement of $\mathbf{w}$. The projections can also be used to decompose the eigenproblem:

$$
(\mathbf{A}-\lambda \mathbf{B}) \mathbf{x}=0 \Leftrightarrow\left\{\begin{aligned}
\mathbf{P}(\mathbf{A}-\lambda \mathbf{B})(\mathbf{Q x}+(\mathbf{I}-\mathbf{Q}) \mathbf{x})=0 \quad \& \\
(\mathbf{I}-\mathbf{P})(\mathbf{A}-\lambda \mathbf{B})(\mathbf{Q} \mathbf{x}+(\mathbf{I}-\mathbf{Q}) \mathbf{x})=0
\end{aligned}\right.
$$

With

$$
\alpha \equiv \frac{\mathbf{w}^{*} \mathbf{A} \mathbf{u}}{\mathbf{w}^{*} \mathbf{u}}, \quad \mathbf{a} \equiv \mathbf{A} \mathbf{u}-\alpha \mathbf{u}, \quad \beta \equiv \frac{\mathbf{w}^{*} \mathbf{B u}}{\mathbf{w}^{*} \mathbf{u}}, \quad \mathbf{b} \equiv \mathbf{B u}-\beta \mathbf{u},
$$

and

$$
\mathbf{u}^{\prime} \equiv\left(\mathbf{I}-\frac{\widetilde{\mathbf{w}} \mathbf{w}^{*}}{\mathbf{w}^{*} \widetilde{\mathbf{w}}}\right) \mathbf{u}=\mathbf{u}-\frac{(\mathbf{u}, \mathbf{w})}{(\widetilde{\mathbf{w}}, \mathbf{w})} \widetilde{\mathbf{w}}
$$

we now see that problem (3.1) is equivalent to the following problem.

$$
\left\{\begin{aligned}
& \text { (a) } \quad \lambda=\frac{\mathbf{w}^{*} \mathbf{A}(\mathbf{u}+\mathbf{z})}{\mathbf{w}^{*} \mathbf{B}(\mathbf{u}+\mathbf{z})} \\
& \text { (b) } \mathbf{z} \perp \tilde{\mathbf{u}} \text { and } \\
&\left.\left(\mathbf{I}-\frac{\tilde{\mathbf{w}} \mathbf{w}^{*}}{\mathbf{w}^{*} \widetilde{\mathbf{w}}}\right)(\mathbf{A}-\lambda \mathbf{B})\right|_{\tilde{\mathbf{u}}^{\perp}} \mathbf{z}=-(\mathbf{a}-\lambda \mathbf{b})-(\alpha-\lambda \beta) \mathbf{u}^{\prime} .
\end{aligned}\right.
$$

The quantities $\alpha, \beta, \mathbf{a}, \mathbf{b}$ and $\mathbf{u}^{\prime}$ can be easily computed. Moreover,

$$
\vartheta=\frac{\alpha}{\beta} \text { and } \mathbf{r}=\mathbf{a}-\vartheta \mathbf{b}=\mathbf{A} \mathbf{u}-\vartheta \mathbf{B u} \text {. }
$$


Algorithm 3.1: The basic algorithm.

Choose a non-trivial $\mathbf{u}$

Repeat:

(a) Select a w.

(b) Compute $\vartheta \equiv \frac{\mathbf{w}^{*} \mathbf{A u}}{\mathbf{w}^{*} \mathbf{B u}}$ and $\mathbf{r} \equiv \mathbf{A u}-\vartheta \mathbf{B u}$.

(c) Stop. Stop if $\mathbf{u}$ and $\vartheta$ are accurate enough.

(d) Select a $\widetilde{\mathbf{u}}$ and a $\widetilde{\mathbf{w}}$.

(e) Solve the correction equation (approximately).

Compute an approximate solution $\mathbf{z}_{1} \perp \widetilde{\mathbf{u}}$

of the correction equation

$$
\left(\mathbf{I}-\frac{\widetilde{\mathbf{w}} \mathbf{w}^{*}}{\mathbf{w}^{*} \widetilde{\mathbf{w}}}\right)(\mathbf{A}-\vartheta \mathbf{B})_{\widetilde{\mathbf{u}}^{\perp}} \mathbf{z}=-\mathbf{r}
$$

(f) Update $\mathbf{u} . \mathbf{q} \equiv \mathbf{u}+\mathbf{z}_{1}, \quad \mathbf{u} \equiv \mathbf{q} /\|\mathbf{q}\|$.

Note that $\mathbf{r} \perp \mathbf{w}$.

If we solve equation (3.5.b) only approximately by replacing $\lambda$ by the approximation $\vartheta$, then we arrive at the following correction equation:

$$
\text { (a) } \mathbf{z}=\left(\mathbf{I}-\frac{\mathbf{u} \widetilde{\mathbf{u}}^{*}}{\widetilde{\mathbf{u}}^{*} \mathbf{u}}\right) \mathbf{z} \quad \text { and } \quad \text { (b) } \quad \mathbf{F}_{p} \mathbf{z}=-\mathbf{r}
$$

where

$$
\mathbf{F}_{p} \equiv\left(\mathbf{I}-\frac{\widetilde{\mathbf{w}} \mathbf{w}^{*}}{\mathbf{w}^{*} \widetilde{\mathbf{w}}}\right)(\mathbf{A}-\vartheta \mathbf{B})\left(\mathbf{I}-\frac{\mathbf{u} \widetilde{\mathbf{u}}^{*}}{\widetilde{\mathbf{u}}^{*} \mathbf{u}}\right)
$$

This suggests the iterative process as described in Algorithm 3.1: compute an approximate solution $\mathbf{z}_{1}$ from equation (3.7.b); update $\mathbf{u}$, (i.e., $\mathbf{u}_{\text {new }}=\mathbf{u}_{\text {old }}+$ $\mathbf{z}_{1}$ ); choose appropriate $\mathbf{w}, \widetilde{\mathbf{u}}$, and $\widetilde{\mathbf{w}}$, in order to satisfy the non-orthogonality conditions for these vectors, and repeat the procedure until the approximate eigenvector $\mathbf{u}$ is accurate enough. If the correction equation is solved exactly, then, according to the following theorems, Theorem 3.2 and Theorem 3.4, we may expect superlinear, and even quadratic convergence, depending on the choices of the $\mathbf{w}, \widetilde{\mathbf{u}}$ and $\widetilde{\mathbf{w}}$.

\subsection{Fast convergence with right eigenvectors.}

In this section $\lambda$ is assumed to be a simple eigenvalue of $\mathbf{A B}^{-1}$ with eigenvector $\mathbf{x} \neq 0$ of the generalized eigenproblem: $(\mathbf{A}-\lambda \mathbf{B}) \mathbf{x}=0$. 
Lemma 3.1. Consider $\mathbf{w}$ and $\widetilde{\mathbf{u}}$ for which $\widetilde{\mathbf{u}}^{*} \mathbf{x} \neq 0$ and $(\mathbf{B x})^{*} \mathbf{w} \neq 0$. Then, the map

$$
\mathbf{F}_{p} \equiv\left(\mathbf{I}-\frac{\mathbf{B} \mathbf{x} \mathbf{w}^{*}}{\mathbf{w}^{*} \mathbf{B} \mathbf{x}}\right)(\mathbf{A}-\lambda \mathbf{B})\left(\mathbf{I}-\frac{\mathbf{x} \widetilde{\mathbf{u}}^{*}}{\widetilde{\mathbf{u}}^{*} \mathbf{x}}\right)
$$

is a bijection from $\widetilde{\mathbf{u}}^{\perp}$ onto $\mathbf{w}^{\perp}$.

Proof. Suppose $\mathbf{y} \perp \widetilde{\mathbf{u}}$ and $\mathbf{F}_{p} \mathbf{y}=0$. Then $(\mathbf{A}-\lambda \mathbf{B}) \mathbf{y}$ is a scalar multiple of $\mathbf{B x}$, and both $\mathbf{B y}$ and $\mathbf{B x}$ belong to the kernel of $\left(\mathbf{A B}^{-1}-\lambda \mathbf{I}\right)^{2}$.

The simplicity of $\lambda$ implies that $\mathbf{B y}$ is a scalar multiple of $\mathbf{B x}$, and hence $\mathbf{y}$ is a multiple of $\mathbf{x}$. The fact that $\mathbf{y} \perp \widetilde{\mathbf{u}}$ and $\widetilde{\mathbf{u}}^{*} \mathbf{x} \neq 0$ implies $\mathbf{y}=0$, which proves the injectivity of $\mathbf{F}_{p}$. An obvious dimension argument implies bijectivity.

The vectors $\widetilde{\mathbf{u}}$ and $\mathbf{w}$ can be chosen differently in each step, and one has to do this carefully in order to ensure the non-orthogonality conditions. One can work with the same vectors at the risk of a breakdown and we will discuss more robust choices in our discussion after the next theorem. We need these vectors to converge in order to be able to make statements on the convergence of the approximations $\mathbf{u}$.

THEOREM 3.2. Assume that the correction equation is solved exactly in each step of Algorithm 3.1. Choose $\widetilde{\mathbf{w}}=\mathbf{B u}$ in each step. Assume that the $\widetilde{\mathbf{u}}$ and $\mathbf{w}$ converge and that both $\widetilde{\mathbf{u}}^{*} \mathbf{x}$ and $\mathbf{w}^{*} \mathbf{B} \mathbf{x}$ have non-trivial limits. Then, if the initial vector $\mathbf{u}$ is close enough to $\mathbf{x}$, the sequence of $\mathbf{u}$ converges in direction to $\mathbf{x}$ and the sequence of $\vartheta=\mathbf{w}^{*} \mathbf{A u} / \mathbf{w}^{*} \mathbf{B u}$ converges to $\lambda$. If the $\mathbf{u}$ converge in direction to $\mathbf{x}$ then the convergence is quadratic.

Proof. Suppose $(\mathbf{A}-\lambda \mathbf{B}) \mathbf{x}=0$ with $\mathbf{x}$ such that $\mathbf{x}=\mathbf{u}+\mathbf{z}$ for $\mathbf{z} \perp \widetilde{\mathbf{u}}$. Then

$$
(\mathbf{A}-\vartheta \mathbf{B}) \mathbf{z}=-(\mathbf{A}-\vartheta \mathbf{B}) \mathbf{u}+(\lambda-\vartheta) \mathbf{B} \mathbf{x}=-\mathbf{r}+(\lambda-\vartheta) \mathbf{B} \mathbf{x} .
$$

Consider the exact solution $\mathbf{z}_{1} \perp \widetilde{\mathbf{u}}$ of the correction equation:

$$
(\mathbf{I}-\mathbf{P})(\mathbf{A}-\vartheta \mathbf{B}) \mathbf{z}_{1}=-(\mathbf{I}-\mathbf{P}) \mathbf{r}
$$

(note that $(\mathbf{I}-\mathbf{P}) \mathbf{r}=\mathbf{r}$, since $\mathbf{w} \perp \mathbf{r}$ ). Since $\mathbf{x}-\left(\mathbf{u}+\mathbf{z}_{1}\right)=\mathbf{z}-\mathbf{z}_{1}$ and $\mathbf{z}=\mathbf{x}-\mathbf{u}$, for quadratic convergence, it suffices to show that

$$
\left\|\mathbf{x}-\left(\mathbf{u}+\mathbf{z}_{1}\right)\right\|=\left\|\mathbf{z}-\mathbf{z}_{1}\right\|=\mathcal{O}\left(\|\mathbf{z}\|^{2}\right) ;
$$

obviously, this relation also implies local convergence.

Multiplying (3.9) by (I - P) and subtracting the result from (3.10) yields

$$
\begin{aligned}
(\mathbf{I}-\mathbf{P})(\mathbf{A}-\vartheta \mathbf{B})\left(\mathbf{z}-\mathbf{z}_{1}\right) \\
=(\lambda-\vartheta)(\mathbf{I}-\mathbf{P}) \mathbf{B} \mathbf{z}+(\lambda-\vartheta)(\mathbf{I}-\mathbf{P}) \mathbf{B u} .
\end{aligned}
$$

Multiplying (3.9) by $\mathbf{w}^{*}$ and using that $\mathbf{r} \perp \mathbf{w}$ leads to

$$
\lambda-\vartheta=\frac{\mathbf{w}^{*}(\mathbf{A}-\vartheta \mathbf{B}) \mathbf{z}}{\mathbf{w}^{*} \mathbf{B} \mathbf{x}} .
$$


Since, by assumption, $\mathbf{w}^{*} \mathbf{B} \mathbf{x}$ has a non-trivial limit, we obtain

$$
\|(\lambda-\vartheta)(\mathbf{I}-\mathbf{P}) \mathbf{B} \mathbf{z}\|=\mathcal{O}\left(\|\mathbf{z}\|^{2}\right)
$$

Because of (3.11), this implies quadratic convergence, if $(\mathbf{I}-\mathbf{P})(\mathbf{A}-\vartheta \mathbf{B})_{\mid \tilde{\mathbf{u}}^{\perp}}$ is non-singular and $(\mathbf{I}-\mathbf{P}) \mathbf{B u}=0$. This last condition holds since $\widetilde{\mathbf{w}}=\mathbf{B u}$. The non-singularity follows from LEMMA 3.1 and the assumption that the sequences of $\mathbf{u}, \mathbf{w}$ and $\widetilde{\mathbf{u}}$ have limits.

We will now make some remarks with respect to the choices for $\widetilde{\mathbf{w}}, \mathbf{w}$, and $\widetilde{\mathbf{u}}$.

REMARK 3.1. If the sequence of approximate eigenvectors $\mathbf{u}$ converges then $\mathbf{A} \mathbf{u} \approx \lambda \mathbf{B u}$, and the choice $\widetilde{\mathbf{w}}=\vartheta \mathbf{A u}+\mathbf{B u}$ also leads to a quadratic convergent process.

REMARK 3.2. If $\widetilde{\mathbf{u}}$ and $\mathbf{w}$ are kept fixed during the iterative process, the sequences of vectors $\widetilde{\mathbf{u}}$ and $\mathbf{w}$ clearly converge. In this case, we are looking for an eigenvector $\mathbf{x}$ with non-trivial component in the direction $\widetilde{\mathbf{u}}$ (the condition $\widetilde{\mathbf{u}}^{*} \mathbf{x} \neq 0$ ) and we hope to find this vector by keeping the residual orthogonal to $\mathbf{w}$. As mentioned earlier, there is no guarantee that all the inner products involved in the projections $\mathbf{P}$ and $\mathbf{Q}$ do not vanish.

Inspection of the arguments in the proof of THEOREM 3.2 reveals that a choice of $\tilde{\mathbf{u}}$ and $\mathbf{w}$ correlated to $\mathbf{u}$, as $\tilde{\mathbf{u}}=\mathbf{w}=\mathbf{u}$ or as $\tilde{\mathbf{u}}=\mathbf{B}^{*} \mathbf{u}, \mathbf{w}=\mathbf{u}$, leads to converging sequences provided that the initial guess $\mathbf{u}$ is sufficiently close to $\mathbf{x}$ and provided that the limit inner products are non-zero $\left(\mathbf{x}^{*} \mathbf{B} \mathbf{x} \neq 0\right)$.

\subsection{Fast convergence with left eigenvectors.}

In the previous section we discussed fast convergence for the case where $\widetilde{\mathbf{w}}$ converges to a (right) eigenvector of $\mathbf{A B}^{-1}$. As we will see in this section, we may also expect fast convergence if the $\mathrm{w}$ converge to a left eigenvector of $\mathbf{A}-\lambda \mathbf{B}$. This approach allows the choice of more natural projections: $\mathbf{P}$ may be selected to be equal to $\mathbf{Q}$ (this will be further exploited in $\S 3.3$ ).

Similar arguments as in the proof of LEMMA 3.1 lead to the following lemma.

LEMMA 3.3. The map

$$
\mathbf{F}_{p} \equiv\left(\mathbf{I}-\frac{\mathbf{x} \mathbf{w}^{*}}{\mathbf{w}^{*} \mathbf{x}}\right)(\mathbf{A}-\lambda \mathbf{B})\left(\mathbf{I}-\frac{\mathbf{x} \widetilde{\mathbf{u}}^{*}}{\widetilde{\mathbf{u}}^{*} \mathbf{x}}\right),
$$

is also a bijection from $\widetilde{\mathbf{u}}^{\perp}$ onto $\mathbf{w}^{\perp}$ if 0 is a simple eigenvalue of the matrix $\mathbf{A}-\lambda \mathbf{B}$.

REMARK 3.3. The scalar $\vartheta$ is a simple eigenvalue of the generalized eigenproblem if $\vartheta$ is a single root of the characteristic polynomial $\operatorname{det}(\mathbf{A}-\lambda \mathbf{B})$, or, equivalently, if $\vartheta$ is a simple eigenvalue of the matrix $\mathbf{A} \mathbf{B}^{-1}$ (recall that $\mathbf{B}$ is non-singular by assumption). However, generally this does not imply that 0 is a simple eigenvalue of the eigenproblem for the matrix $\mathbf{A}-\vartheta \mathbf{B}$. Although the geometrical multiplicity is one, the algebraic multiplicity can be larger. If the geometric and the algebraic multiplicity coincide, for instance if both $\mathbf{A}$ and $\mathbf{B}$ are self-adjoint $\left(\mathbf{A}^{*}=\mathbf{A}\right.$ and $\left.\mathbf{B}^{*}=\mathbf{B}\right)$, and $\vartheta$ is real, then 0 is a simple eigenvalue of $\mathbf{A}-\vartheta \mathbf{B}$ when $\vartheta$ is a simple eigenvalue of $\mathbf{A B}^{-1}$.

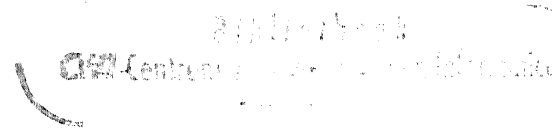


Theorem 3.4. Assume that 0 is a simple eigenvalue of $\mathbf{A}-\lambda \mathbf{B}$. Assume that, in each step of Algorithm 3.1, the correction equation is solved exactly. Assume the $\tilde{\mathbf{u}}$ 's, w's and $\tilde{\mathbf{w}}$ 's converge, $\widetilde{\mathbf{u}}^{*} \mathbf{u}, \widetilde{\mathbf{w}}^{*} \mathbf{w}$ and $\mathbf{w}^{*} \mathbf{B u}$ have non-trivial limits and $(\mathbf{A}-\vartheta \mathbf{B})^{*} \mathbf{w}$ converges towards 0 . Then $\mathbf{u}$ converges in direction to $\mathbf{x}$ if the initial vector $\mathbf{u}$ is close enough to $\mathbf{x}$. If $\mathbf{u}$ converges in direction to $\mathbf{x}$ then the convergence is superlinear.

Proof. The assumptions and (3.12) imply that $|\lambda-\vartheta|=o(\|\mathbf{z}\|)$ and, as in the proof of Theorem 3.2, superlinear convergence follows from (3.11).

REMARK 3.4. If both $\mathbf{A}$ and $\mathbf{B}$ are self adjoint and $\lambda \in \mathbb{R}$ then, for the choice $\mathbf{w}=\mathbf{u}$, we have that $\mathbf{w}^{*}(\mathbf{A}-\lambda \mathbf{B})=-\mathbf{z}^{*}(\mathbf{A}-\lambda \mathbf{B})$. Therefore, in this "symmetric case", we have that

$$
|\lambda-\vartheta|=\mathcal{O}\left(\|\mathbf{z}\|^{2}\right)
$$

which implies quadratic convergence for any choice of $\tilde{\mathbf{w}}$.

REMARK 3.5. More general, we obtain quadratic convergence if we select our $\mathbf{w}$ to converge to the left eigenvector of $\mathbf{A}-\lambda \mathbf{B}$, by an obvious adjoint version of Algorithm 3.1, that is, with $\mathbf{r}_{\ell} \equiv(\mathbf{A}-\vartheta \mathbf{B})^{*} \mathbf{w}$, solve exactly the adjoint correction equation:

$$
\mathbf{z}_{\ell} \perp \tilde{\mathbf{w}} \operatorname{such} \text { that } \mathbf{z}_{\ell}^{*}\left(\mathbf{I}-\frac{\widetilde{\mathbf{w}} \mathbf{w}^{*}}{\mathbf{w}^{*} \tilde{\mathbf{w}}}\right)(\mathbf{A}-\vartheta \mathbf{B})\left(\mathbf{I}-\frac{\mathbf{u} \tilde{\mathbf{u}}^{*}}{\widetilde{\mathbf{u}}^{*} \mathbf{u}}\right)=-\mathbf{r}_{\ell}^{*}
$$

and update $\mathbf{w}$ similar to $\mathbf{u}: \mathbf{w}^{\prime} \equiv \mathbf{w}+\mathbf{z}_{\ell}, \mathbf{w} \equiv \mathbf{w}^{\prime} /\left\|\mathbf{w}^{\prime}\right\|$. Note that $\mathbf{r}_{\ell} \perp \mathbf{u}$.

With this approach we also have converging $\mathbf{u}, \mathbf{w}, \widetilde{\mathbf{u}}$, and $\widetilde{\mathbf{w}}$, if the initial $\mathbf{u}$ is close enough to $\mathbf{x}$, the initial $\mathbf{w}$ is close enough to the left eigenvector of $\mathbf{A}-\lambda \mathbf{B}$, and $\tilde{\mathbf{u}}, \tilde{\mathbf{w}}$ are fixed or $\tilde{\mathbf{u}}=\mathbf{w}, \widetilde{\mathbf{w}}=\mathbf{u}$, and the non-zero conditions on the inner products are fulfilled. We obtain cubic convergence if, in addition, we choose $\widetilde{\mathbf{w}}=\mathrm{Bu}$.

\subsection{The choice of the projections $\mathbf{P}$ and $\mathbf{Q}$.}

If $\mathbf{w} \neq \tilde{\mathbf{u}}$ then the domain space $\tilde{\mathbf{u}}^{\perp}$ of the map $\mathbf{F}_{p}$ in (3.8) differs from the image space $\mathbf{w}^{\perp}$ of this map $\mathbf{F}_{p}$. Powers of $\mathbf{F}_{p}$ cannot be formed, and to solve equation (3.7) by some Krylov subspace method, we need a "preconditioner" $\mathbf{K}$ that maps the image space $\mathbf{w}^{\perp}$ bijectively onto the domain space $\widetilde{\mathbf{u}}{ }^{\perp}$ : then we can apply Krylov subspace methods to the preconditioned system (see $\S 7.1$ ). In other words, for unpreconditioned Krylov subspace methods, it is desirable to have $(\mathbf{I}-\mathbf{P})(\mathbf{I}-\mathbf{Q})=\mathbf{I}-\mathbf{Q}$, or, equivalently, $\widetilde{\mathbf{u}}=\mathbf{w}$.

The choice $\tilde{\mathbf{w}}=\mathrm{Bu}, \tilde{\mathbf{u}}=\mathbf{w}$ leads to a fast converging process in which the correction equation requires a solution in the space that also contains the residual.

The choice $\tilde{\mathbf{w}}=\mathbf{u}, \tilde{\mathbf{u}}=\mathbf{w}$ and $\mathbf{w}$ some approximate left eigenvector leads to fast convergence (cf. $\S 3.2$ ), and the projections $\mathbf{P}$ and $\mathbf{Q}$ coincide. If 0 is a simple eigenvalue of $\mathbf{A}-\lambda \mathbf{B}$, and $\mathbf{u}$ and $\mathbf{w}$ converge to the associated right and left eigenvector, respectively, then $\widetilde{\mathbf{w}}^{*} \mathbf{w}=\mathbf{u}^{*} \mathbf{w}$ has a non-trivial limit value. 
By embedding the correction equation into the entire space $\mathbb{C}^{n}$, we also circumvent the problem of having a domain space that differs from the image space: in the whole space, we trivially have the same image and domain space. For instance, the choice $\tilde{\mathbf{w}}=\mathbf{B u}, \tilde{\mathbf{u}}=\mathbf{B}^{*} \mathbf{w}$, implies $\mathbf{B Q}=\mathbf{P B}$. Hence, $(\mathbf{I}-\mathbf{P}) \mathbf{B}=(\mathbf{I}-\mathbf{P}) \mathbf{B}(\mathbf{I}-\mathbf{Q})$. Since $\mathbf{r}=(\mathbf{I}-\mathbf{P}) \mathbf{r}$, the solution $\mathbf{z}$ of problem (3.7) is precisely $\mathbf{z}=(\mathbf{I}-\mathbf{Q}) \tilde{\mathbf{z}}$ where $\tilde{\mathbf{z}} \in \mathbb{C}^{n}$ is the solution of the equation

$$
\left(\mathbf{A}_{p}-\vartheta \mathbf{B}\right) \tilde{\mathbf{z}}=-\mathbf{r} \text { where } \mathbf{A}_{p} \equiv\left(\mathbf{I}-\frac{\mathbf{B u} \mathbf{w}^{*}}{\mathbf{w}^{*} \mathbf{B} \mathbf{u}}\right) \mathbf{A}\left(\mathbf{I}-\frac{\mathbf{u} \mathbf{w}^{*} \mathbf{B}}{\mathbf{w}^{*} \mathbf{B} \mathbf{u}}\right)
$$

The embedded equation in (3.16) is non-singular if the equation in (3.7) is nonsingular, $\vartheta \neq 0$ and $\beta \neq 0$ (or, equivalently, $\mathbf{w}^{*} \mathbf{B u} \neq 0$ ).

The choice $\widetilde{\mathbf{w}}=\mathbf{B u}, \widetilde{\mathbf{u}}=\mathbf{B}^{*} \mathbf{w}$, leads to a fast converging process and the correction equation can be embedded in the entire space. If, in addition, $\mathbf{w}=\mathbf{u}$ and $\mathbf{B}$ is self-adjoint then $\mathbf{P}^{*}=\mathrm{Q}$. With $\mathbf{w}=\tilde{\mathbf{w}}=\mathbf{B u}$ and $\tilde{\mathbf{u}}=\mathbf{B}^{*} \mathbf{B u}$ the inner product $\widetilde{\mathbf{w}}^{*} \mathbf{w}=\widetilde{\mathbf{u}}^{*} \mathbf{u}\left(=\|\mathbf{B u}\|^{2}\right)$ is non-zero. This is also the case if $\mathbf{B}$ is positive definite and $\mathbf{w}=\mathbf{u}$.

\subsection{Equivalent formulations for the correction equation.}

The dimension of the problem defined by the correction equation (3.7) is smaller than the dimension of the space of the eigenproblem: the projections are used to restrict the image space and domain space to a space of co-dimension 1. As is stated by the following theorem, the projections can also be used for an equivalent formulation in a space of larger dimension. This equivalent augmented formulation may be useful for the construction of preconditioners for the correction equation (3.7) (cf. §7.1). For similar reasons [9], such an augmented equation also appears in the theory for numerical continuation methods for nonlinear equations (cf., e.g., [11]).

THEOREM 3.5. Equation (3.7) is equivalent to

$$
\left[\begin{array}{cc}
\mathbf{A}-\vartheta \mathbf{B} & \tilde{\mathbf{w}} \\
\tilde{\mathbf{u}}^{*} & 0
\end{array}\right]\left[\begin{array}{l}
\mathbf{z} \\
\varepsilon
\end{array}\right]=\left[\begin{array}{r}
-\mathbf{r} \\
0
\end{array}\right]
$$

that is, $\mathbf{z}$ is a solution of (3.17) if and only if $\mathbf{z}$ is a solution of (3.7).

ProOF. Obviously, equation (3.17) implies that $\tilde{\mathbf{u}}^{*} \mathbf{z}=0$, and this holds if and only if $\mathbf{z} \perp \tilde{\mathbf{u}}$.

Since $\mathbf{r} \perp \mathbf{w}$, the first block coordinate $(\mathbf{A}-\vartheta \mathbf{B}) \mathbf{z}+\varepsilon \tilde{\mathbf{w}}=-\mathbf{r}$ in $(3.17)$ is equivalent to

$$
\left\{\begin{array}{l}
\left(\mathbf{I}-\frac{\tilde{\mathbf{w}} \mathbf{w}^{*}}{\mathbf{w}^{*} \tilde{\mathbf{w}}}\right)((\mathbf{A}-\vartheta \mathbf{B}) \mathbf{z}+\varepsilon \tilde{\mathbf{w}})=-\mathbf{r} \text { and } \\
\mathbf{w}^{*}((\mathbf{A}-\vartheta \mathbf{B}) \mathbf{z}+\varepsilon \tilde{\mathbf{w}})=0 .
\end{array}\right.
$$

Since $\left(I-\frac{\widetilde{\mathbf{w}} \mathbf{w}^{*}}{\mathbf{w}^{*} \mathbf{w}}\right) \tilde{\mathbf{w}}=0$, we see that the first equation in $(3.18)$ is equivalent to (3.7). The second equation determines $\varepsilon\left(=-\mathbf{w}^{*}(\mathbf{A}-\vartheta \mathbf{B}) \mathbf{z} / \mathbf{w}^{*} \tilde{\mathbf{w}}\right)$. 
REMARK 3.6. In (3.7), the vectors $\mathbf{u}$ and $\mathbf{w}$ play a role. These vectors do not occur in equation (3.17). However, they enter through $\mathbf{r}=(\mathbf{A}-\vartheta \mathbf{B}) \mathbf{u}$ and the condition $\mathbf{r} \perp \mathbf{w}$. For the construction of incomplete factorizations that may serve as preconditioners it may be advantageous to reorder the equations in (3.17).

Using the fact that $\mathbf{r}=(\mathbf{A}-\vartheta \mathbf{B}) \mathbf{u},(3.17)$, and therefore (3.7), can be solved exactly in terms of the solution $\mathbf{t}$ of the equation $(\mathbf{A}-\vartheta \mathbf{B}) \mathbf{t}=\widetilde{\mathbf{w}}$ :

THEOREM 3.6. The solution $\mathbf{z}$ of (3.7) (and of (3.17)) is given by

$$
\begin{aligned}
\mathbf{z} & =(\mathbf{A}-\vartheta \mathbf{B})^{-1}(-\mathbf{r}+\widetilde{\mathbf{w}}) \\
& =-\mathbf{u}+\varepsilon(\mathbf{A}-\vartheta \mathbf{B})^{-1} \widetilde{\mathbf{w}} \quad \text { with } \quad \varepsilon=\frac{\widetilde{\mathbf{u}}^{*} \mathbf{u}}{\widetilde{\mathbf{u}}^{*}(\mathbf{A}-\vartheta \mathbf{B})^{-1} \widetilde{\mathbf{w}}} .
\end{aligned}
$$

Proof. With $\mathbf{z}$ as in (3.19), it is easily verified that $\widetilde{\mathbf{u}} \perp \mathbf{z}$ and $(\mathbf{A}-\vartheta \mathbf{B}) \mathbf{z}=$ $-\mathbf{r}+\varepsilon \widetilde{\mathbf{w}}$. Since $\mathbf{r} \perp \mathbf{w}$ it follows that (3.7) holds.

For $\widetilde{\mathbf{w}}=\mathbf{B u}$, this theorem gives a relation to Rayleigh Quotient Iteration. In particular, we see that Algorithm 3.1 leads to cubical convergent sequences if $\mathbf{B}=\mathbf{I}, \mathbf{A}$ is symmetric, $\widetilde{\mathbf{u}}=\mathbf{w}=\widetilde{\mathbf{w}}=\mathbf{u}$, and if the correction equation is solved exactly, which is in agreement with observations made in $\S 3.2$. In $\S 4$ we use the approximate solution $\mathbf{z}$ to expand a subspace in which we search for new approximations for the eigenvector $\mathbf{x}$. This subspace will contain the current approximation $\mathbf{u}$ and expanding it by $\mathbf{z}$ (as in (3.19)) is equivalent to expanding it by $(\mathbf{A}-\vartheta \mathbf{B})^{-1} \widetilde{\mathbf{w}}$. For $\widetilde{\mathbf{w}}=\mathbf{B u}$, this reveals a relation between the method to be discussed in $\S 4$ and Shift-and-Inverse Arnoldi; see Discussion 4.1.

\section{Projections on subspaces for approximating the eigenproblem.}

In the previous sections we have used projections $\mathbf{P}$ and $\mathbf{Q}$ defined with only single vectors $\widetilde{\mathbf{w}}$ and $\widetilde{\mathbf{u}}$. The older approximations were discarded, but following Davidson's approach, we will now take also these approximations into account, in order to prevent a search in a subspace that has already been explored. In this approach we store vectors that have been computed in previous steps, and we update $\mathbf{u}$ in the best way that is possible using all these vectors. The general Jacobi-Davidson (JD) algorithm, Algorithm 4.2, that we introduce in this section, is the basic Algorithm 3.1 with an improved updating strategy for the approximate eigenvector $\mathbf{u}$.

At the beginning of the $k$ th step we have two sets of linearly independent vectors $\mathbf{v}_{1}, \ldots, \mathbf{v}_{k}$ and $\mathbf{w}_{1}, \ldots, \mathbf{w}_{k} . \mathbf{V}_{k}$ is the matrix with the $\mathbf{v}_{i}$ as columns $(i=1, \ldots, k), \mathcal{V}_{k}=\operatorname{span}\left(\mathbf{V}_{k}\right)$, i.e., $\mathcal{V}_{k}$ is the subspace spanned by the columns $\mathbf{v}_{i}$ of $\mathbf{V}_{k}$. We use a similar notation $\mathbf{W}_{k}$ and $\mathcal{W}_{k}$ in relation to the $\mathbf{w}_{i}$. Then we construct our new approximating eigenvector $\mathbf{u}$, our new approximating eigenvalue $\vartheta$ by solving the projected eigenproblem:

$$
\mathbf{u} \in \mathcal{V}_{k}, \quad \vartheta \in \mathbb{C} \quad \text { that } \mathbf{r} \equiv \mathbf{A} \mathbf{u}-\vartheta \mathbf{B u} \perp \mathcal{W}_{k} .
$$

Since this projected problem is equivalent to finding a $y \in \mathbb{C}^{k}, \vartheta \in \mathbb{C}$ such that

$$
\mathbf{W}_{k}^{*} \mathbf{A V}_{k} y-\vartheta \mathbf{W}_{k}^{*} \mathbf{B V}_{k} y=0 \text { and } \quad \mathbf{u}=\mathbf{V}_{k} y
$$


Algorithm 4.2: The Jacobi-Davidson algorithm.

Choose a non-trivial $\mathbf{v}$ and a non-trivial $\mathbf{w}$.

Set $\mathbf{V}=[\mathbf{v}], \mathbf{W}=[\mathbf{w}], k=0$.

Repeat:

(a) Solve the projected eigenproblem.

Compute non-trivial solutions $y \in \mathbb{C}^{k+1}$ and associated $\vartheta \in \mathbb{C}$ of the projected eigenproblem

$$
\mathbf{W}^{*} \mathbf{A V} y-\vartheta \mathbf{W}^{*} \mathbf{B V} y=0 .
$$

(b) Select approximating eigenvector and eigenvalue. Select a solution $y$ and associated Petrov value $\vartheta$.

Compute the Petrov vector $\mathbf{u} \leftarrow \mathbf{V} y$ and the residual $\mathbf{r} \leftarrow \mathbf{A u}-\vartheta \mathbf{B u}$.

(c) Stop. Stop if $\mathbf{u}$ and $\vartheta$ are accurate enough.

(d) Select a $\mathbf{w}$ in $\operatorname{span}(\mathbf{W})$ and select $\widetilde{\mathbf{u}}$ and $\widetilde{\mathbf{w}}$.

(e) Solve the correction equation (approximately). Compute an approximate solution $\mathbf{z}_{1} \perp \widetilde{\mathbf{u}}$ of the correction equation

$$
\left.\left(\mathbf{I}-\frac{\widetilde{\mathbf{w}} \mathbf{w}^{*}}{\mathbf{w}^{*} \widetilde{\mathbf{w}}}\right)(\mathbf{A}-\vartheta \mathbf{B})\right|_{\widetilde{\mathbf{u}}^{\perp}} \mathbf{z}=-\mathbf{r} .
$$

(f) Restart. $k \leftarrow \operatorname{dim}(\operatorname{span}(\mathbf{V}))$.

$$
\text { If } k \text { is too large: }
$$

select an $\ell<k$, select $k \times \ell$ matrices $R_{V}$ and $R_{W}$,

compute $\mathbf{V} \leftarrow \mathbf{V} R_{V}, \mathbf{W} \leftarrow \mathbf{W} R_{W}, k \leftarrow \ell$.

(g) Expand the search subspace.

Select a $\mathbf{v} \in \operatorname{span}\left(\mathbf{V}, \mathbf{z}_{1}\right) \backslash \operatorname{span}(\mathbf{V})$ and $\mathbf{V} \leftarrow[\mathbf{V}, \mathbf{v}]$.

(h) Expand the test subspace.

Select a $\widetilde{\mathbf{v}}, \widetilde{\mathbf{v}} \notin \operatorname{span}(\mathbf{W})$ and $\mathbf{W} \leftarrow[\mathbf{W}, \widetilde{\mathbf{v}}]$.

we only have to solve a small generalized eigenproblem of size $k\left(\mathbf{W}_{k}^{*} \mathbf{A V}_{k}\right.$ and $\mathbf{W}_{k}^{*} \mathbf{B} \mathbf{V}_{k}$ are $k \times k$ matrices) and compute $\mathbf{u}$ as a linear combination of the $\mathbf{v}_{i}$.

DEFINITION 4.1. Since we look for the new approximate eigenvector in the subspace $\mathcal{V}_{k}$, we call $\mathcal{V}_{k}$ the search subspace. The subspace $\mathcal{W}_{k}$ is used in the projection to define an approximating problem. Analogously to the situation with Petrov-Galerkin methods, we will refer to $\mathcal{W}_{k}$ as the test subspace. We use the words Petrov value for the approximate eigenvalue $\vartheta$ and Petrov vector for the approximate eigenvector $\mathbf{u}=\mathbf{V}_{k} y(\vartheta, y$ solution of (4.2); see also $\S 5.1)$.

The approximate solution $\mathbf{z}_{1}$ of the correction equation (3.7) is used to expand 
the $k$-dimensional search subspace $\mathcal{V}_{k}$ to the subspace $\mathcal{V}_{k+1}$ of dimension $k+1$. Next, we select (or construct) a new $\mathbf{v}_{k+1}$ vector in the set of $\mathbf{v}_{i} ; \mathbf{v}_{k+1}$ belongs to $\mathcal{V}_{k+1}$, and is linearly independent of $\mathbf{v}_{1}, \ldots, \mathbf{v}_{k}$. Finally, we construct a new $\mathbf{w}_{k+1}$. Algorithm 4.2 shows an algorithmical formulation of this strategy. In this formulation, we have suppressed the index $k$ and we have included the possibility of reducing the search subspace and the test subspace (step (f)).

By expanding our subspace, we may expect better global convergence properties. But, moreover, we also may expect better local convergence. The choice $\widetilde{\mathbf{w}}=\mathbf{B u}$ (cf. Theorem 3.2) leads to 'superquadratic' convergence (in analogy with the 'superlinear' convergence for Krylov subspace methods): the test subspace increases and will contain better approximations for the left eigenvector, which also leads to better approximations for $\vartheta$ (cf. (3.12), since in this case, by (4.1), for $\mathbf{w}$ we may take any vector in $\mathcal{W}_{k}$ ).

Any of the substeps (a)-(h) in Algorithm 4.2 can be performed in a number of ways. Different choices in any of the substeps (b), (d), (f) and (h) lead to different sequences of approximating eigenvectors and eigenvalues. The specific choice of the basis vectors $\mathbf{v}_{k+1}$ and $\mathbf{w}_{k+1}$ in (g) and (h) affect the computational complexity and the stability of the algorithm. The choice of the subspaces $\mathcal{V}_{k}$ (through (d), (e) and the restart strategy in (f)) and $\mathcal{W}_{k}$ (in (h)) affects the convergence behavior, while the choices of the basis of these subspace (in (g) and (h)) affect the computational complexity and the stability. In this paper we will comment on the following substeps: on (a) in $\S 6$; (d) in $\S 3$; (e) in $\S 7$ (and 3); $(\mathrm{g})$ and $(\mathrm{h})$ in $\S 5$. For $(\mathrm{b})$ and $(\mathrm{f})$ we refer to [10].

Discussion 4.1. In order to keep our arguments simple, we will assume in this discussion that $\mathbf{B}=\mathbf{I}$. Furthermore, we will not consider restarts, and in Algorithm 4.2, we will take $\mathbf{W}=\mathbf{V}, \widetilde{\mathbf{w}}=\mathbf{u} . \mathbf{v}_{\mathbf{1}}$ is the initial vector $\mathbf{v}$.

THEOREM 3.6 indicates a relation to Shift-and-Invert Arnoldi (cf., e.g., [24, ALG. 8.1]). For this algorithm the user has to provide a complex value $\mu$ and the algorithm locates the approximate eigenvalue nearest to $\mu$ with associated eigenvector. The algorithm is based on Arnoldi's method for $(\mathbf{A}-\mu \mathbf{I})^{-1}$. If we take in step (e) of Algorithm 4.2 for $\mathbf{z}_{1}$ the exact solution of the correction equation (in which $\vartheta$ is replaced by $\mu$ ), then our search subspace $\mathcal{V}_{k}$ coincides with the Krylov subspace $\mathcal{K}_{k}\left((\mathbf{A}-\mu \mathbf{I})^{-1} ; \mathbf{v}_{1}\right)$ (cf.Theorem 3.6), i.e., the 'search subspace' of Shift-and-Invert Arnoldi. Therefore, if we orthogonalize in step (g) of Algorithm 4.2 against the previously constructed $\mathbf{V}$, then (except for some scalar multiple) the orthonormal basis vectors for both algorithms coincide and both algorithms produce the same approximate eigenpair. Since, Arnoldi's method exploits the Hessenberg structure of the projected matrix (here the projection of $\left.(\mathbf{A}-\mu \mathbf{I})^{-1}\right)$, Shift-and-Invert Arnoldi is more efficient than JD in this special case.

Replacing the 'target value' $\mu$ by the currently optimal approximate eigenvalue $\vartheta$ in Shift-and-Invert Arnoldi may be expected to lead to faster convergence but it affects also the efficiency: the projected matrix will be dense and in exact arithmetic both methods lead to the same results at the same costs. Since it may be expensive to work with $(\mathbf{A}-\vartheta \mathbf{I})^{-1}$ (i.e., to solve equations as $(\mathbf{A}-\vartheta \mathbf{I}) \mathbf{z}=\mathbf{r}$ 
exactly) in each step, one might prefer suitable approximations $\mathbf{K}$ of $\mathbf{A}-\vartheta \mathbf{I}$ or of $\mathbf{A}-\mu \mathbf{I}$ (preconditioners; cf. $[5,8,17,18,20]$ ). Then, in $\mathbf{J D}$, the search subspace is expanded by some appropriate linear combination of $\mathbf{K}^{-1}(\mathbf{A}-\vartheta \mathbf{I}) \mathbf{u}$ and $\mathbf{K}^{-1} \mathbf{u}$ while the Shift-and-Invert Arnoldi, properly adapted, will expand by $\mathbf{K}^{-1} \mathbf{u}$ only. However, in view of the success of Davidson's method the component $\mathbf{K}^{-1}(\mathbf{A}-\vartheta \mathbf{I}) \mathbf{u}$ is apparently important.

\section{The search subspace and the test subspace.}

For the approximation of the eigenvector $\mathbf{x}$ in a relatively small number $k$ of sweeps, we wish to construct a search subspace $\mathcal{V}_{k}$ that makes a (very) small angle with the vector $\mathbf{x}$. The choice $\mathcal{V}_{k}=\operatorname{span}(\mathbf{x})$ is optimal, but impractical. We will first consider the construction of suitable test subspaces and after that we will consider the search subspace.

\subsection{The test subspace.}

We will be interested in the non-trivial solutions $\mathbf{u}, \vartheta$ of the projected eigenproblem (4.1). The test subspace determines the way of projection, e.g., orthogonal with $\mathcal{W}_{k}=\mathcal{V}_{k}$, or oblique with other choices.

Different choices can be made for $\mathcal{W}_{k}$. Besides the choice for $\mathcal{W}_{k}$ as a subspace of approximating left eigenvectors (cf. §5.1.3), the following three other choices are obvious and allow for a familiar interpretation that we will discuss (in $\S 5.1 .1$ and 5.1.2): $\mathcal{W}_{k}=\mathcal{V}_{k}, \mathcal{W}_{k}=\mathbf{A} \mathcal{V}_{k}$, and $\mathcal{W}_{k}=\mathbf{B} \mathcal{V}_{k}$. These interpretations involve Ritz vectors and harmonic Ritz vectors: if $\mathbf{A}$ is an $n \times n$ matrix and $\mathcal{V}$ is a $k$ dimensional subspace of $\mathbb{C}^{k}$ then a non-zero vector $\mathbf{u}$ in $\mathcal{V}$ and a scalar $\vartheta \in \mathbb{C}$ are called a Ritz vector and Ritz value, respectively, of $\mathbf{A}$ with respect to $\mathcal{V}$ if $\mathbf{A} \mathbf{u}-\vartheta \mathbf{u} \perp \mathcal{V}$. They are referred to as harmonic Ritz vector and harmonic Ritz value, respectively, of $\mathbf{A}$ with respect to $\mathcal{V}$ if $\mathbf{A u}-\vartheta \mathbf{u} \perp \mathbf{A} \mathcal{V}[22,29]$. Note that $\mathbf{u}$ is a harmonic Ritz vector of $\mathbf{A}$ with respect to $\mathcal{V}$ if and only if $\mathbf{A} \mathbf{u}$ is a Ritz vector of $\mathbf{A}^{-1}$ with respect to the subspace $\mathbf{A} \mathcal{V}$. Note that, although we compute Ritz vectors of $\mathbf{A}^{-1}$, we never have to compute any inverse because of the search space $\mathbf{A} \mathcal{V}$.

In computations, sequences $\left(\mathcal{V}_{k}\right)$ of subspaces of increasing dimension are constructed, where the $k$-dimensional subspace $\mathcal{V}_{k}$ is a subset of the $k+1$ dimensional subspace $\mathcal{V}_{k+1}$. When $\mathbf{A}$ is normal (or close to normal) then the extremal Ritz values usually exhibit some regular convergence behavior towards extremal eigenvalues, while the in absolute value smallest harmonic Ritz values usually show a regular convergence behavior towards the in absolute value smallest eigenvalues (for this property for symmetric matrices, see [22]). These properties may be used for the selection of the approximations of interest, especially for restarting purposes.

Once we have $\mathbf{V}_{k}$, any of the matrices $\mathbf{A V}_{k}$ and $\mathbf{B V}_{k}$ can easily be computed, and probably will be computed anyway to facilitate the computation of the $k \times k$ matrices $\mathbf{W}_{k}^{*} \mathbf{A V}_{k}$ and $\mathbf{W}_{k}^{*} \mathbf{B} \mathbf{V}_{k}$.

In the next subsections we will discuss some choices for $\mathbf{W}$ for the important case that $\mathbf{B}$ is nonsingular. 


\subsubsection{B is non-singular.}

(a) $\mathbf{W}=\mathbf{A V}$. The solutions of (4.1) with $\mathcal{W}_{k}=\mathbf{A} \mathcal{V}_{k}$ correspond to harmonic Ritz values of $\mathbf{A B}^{-1}$ with respect to the subspace $\widetilde{\mathbf{V}} \equiv \mathbf{B V}$. That means that for $\widetilde{\mathbf{u}}=\mathbf{B} \mathbf{u}$ with $\mathbf{u} \in \mathbf{V}$ the requirement

$$
\mathbf{A B}^{-1} \widetilde{\mathbf{u}}-\vartheta \widetilde{\mathbf{u}} \perp \mathbf{A B}^{-1} \widetilde{\mathbf{V}}
$$

is equivalent to (4.1) with $\mathcal{W}_{k}=\mathbf{A} \mathcal{V}_{k}$. Note again that, although the inverse of $\mathbf{B}$ appears, operations with $\mathbf{B}$ are never necessary, for instance $\mathbf{B}^{-1} \widetilde{\mathbf{u}}=\mathbf{u}$.

(b) $\mathbf{W}=\mathbf{B V}$. The solution of (4.1) with $\mathcal{W}_{k}=\mathbf{B} \mathcal{V}_{k}$ corresponds to Ritz values of $\mathbf{A B}^{-1}$ with respect to the subspace $\tilde{\mathbf{V}} \equiv \mathbf{B V}$ in the following sense. For $\widetilde{\mathbf{u}}=\mathbf{B u}$ with $\mathbf{u} \in \mathbf{V}$ we have that

$$
\mathbf{A B}^{-1} \widetilde{\mathbf{u}}-\vartheta \widetilde{\mathbf{u}} \perp \widetilde{\mathbf{V}}
$$

is equivalent to (4.1) with $\mathcal{W}_{k}=\mathbf{B} \mathcal{V}_{k}$.

\subsection{2 $\mathbf{B}$ is positive definite.}

If $\mathbf{B}$ is positive definite then we can exploit a $\mathbf{B}$-inner product and associated orthogonality.

(a) $\mathbf{W}=\mathbf{V}$. The solutions of (4.1) with $\mathcal{W}_{k}=\mathcal{V}_{k}$ correspond to:

(a1) Ritz values of $\mathbf{A B}^{-1}$ with respect to the $\mathbf{B}^{-1}$-inner product and the subspace $\widetilde{\mathbf{V}} \equiv \mathbf{B V}$, in the following way. For $\widetilde{\mathbf{u}}=\mathbf{B u}$ with $\mathbf{u} \in \mathbf{V}$ it follows that

$$
\mathbf{A B}^{-1} \widetilde{\mathbf{u}}-\vartheta \widetilde{\mathbf{u}} \perp_{\mathbf{B}^{-1}} \widetilde{\mathbf{V}}
$$

is equivalent to (4.1) with $\mathcal{W}_{k}=\mathcal{V}_{k}$.

(a2) Ritz values of $\mathbf{B}^{-\frac{1}{2}} \mathbf{A} \mathbf{B}^{-\frac{1}{2}}$ with respect to the subspace $\tilde{\mathbf{V}} \equiv \mathbf{B}^{\frac{1}{2}} \mathbf{V}$, as follows: for $\widetilde{\mathbf{u}}=\mathbf{B}^{\frac{1}{2}} \mathbf{u}$ with $\mathbf{u} \in \mathbf{V}$ we have that

$$
\mathbf{B}^{-\frac{1}{2}} \mathbf{A B}^{-\frac{1}{2}} \widetilde{\mathbf{u}}-\vartheta \widetilde{\mathbf{u}} \perp \tilde{\mathbf{V}}
$$

is equivalent to (4.1) with $\mathcal{W}_{k}=\mathcal{V}_{k}$

(b) $\mathbf{W}=\mathbf{A V}$. The solutions of (4.1) with $\mathcal{W}_{k}=\mathbf{A} \mathcal{V}_{k}$ correspond to harmonic Ritz values of $\mathbf{B}^{-\frac{1}{2}} \mathbf{A} \mathbf{B}^{-\frac{1}{2}}$ with respect to the $\mathbf{B}$-inner product and the subspace $\widetilde{\mathbf{V}} \equiv \mathbf{B}^{\frac{1}{2}} \mathbf{V}$ in the following way. For $\widetilde{\mathbf{u}}=\mathbf{B}^{\frac{1}{2}} \mathbf{u}$ with $\mathbf{u} \in \mathbf{V}$ we have that

$$
\mathbf{B}^{-\frac{1}{2}} \mathbf{A B}^{-\frac{1}{2}} \widetilde{\mathbf{u}}-\vartheta \widetilde{\mathbf{u}} \perp_{\mathbf{B}} \mathbf{B}^{-\frac{1}{2}} \mathbf{A} \mathbf{B}^{-\frac{1}{2}} \widetilde{\mathbf{V}}
$$

is equivalent to (4.1) with $\mathcal{W}_{k}=\mathbf{A} \mathcal{V}_{k}$.

\subsubsection{The test subspace as a search subspace.}

As explained in $§ 3.2$, we obtain fast convergence if the $\mathbf{w}$ converge to the left eigenvector. Therefore, the construction of $\mathcal{W}_{k}$ similar to $\mathcal{V}_{k}$ is straightforward: solve both the correction equation (3.7) and the adjoint correction equation (3.15) approximately (the Bi-CG method seems to fit quite naturally in this approach), and extend the search subspace and the test subspace by the computed corrections. Then take $\mathbf{w}=\mathbf{W}_{k} y$, where $y$ is the left eigenvector of the projected eigenproblem associated with $\vartheta$. 


\subsection{On the choice of $\mathbf{w}$.}

We have discussed choices for $\mathbf{w}, \widetilde{\mathbf{w}}$ and $\widetilde{\mathbf{u}}$ in $\S 3$. However, these choices cannot be completely independent from the subspaces $\mathcal{V}_{k}$ and $\mathcal{W}_{k}$. For instance, w should be in $\mathcal{W}_{k}$, and should not be orthogonal to $\tilde{\mathbf{w}}$.

For a number of choices (cf. §3.3), w should not be orthogonal to $\mathbf{u}$ as well. In particular, $\mathbf{u}$ should not be orthogonal to $\mathcal{W}_{k}$ and it is quite natural to require that the subspaces $\mathcal{V}_{k}$ and $\mathcal{W}_{k}$ are not mutually orthogonal.

If $\mathbf{W}=\mathbf{B V}$, then the choices $\widetilde{\mathbf{w}}=\mathbf{w}=\mathbf{B u}$ and $\widetilde{\mathbf{u}}=\mathbf{B}^{*} \mathbf{w}$ are obvious. The same remark applies to the combination of $\mathbf{W}=\mathbf{A V}, \widetilde{\mathbf{w}}=\mathbf{w}=\mathbf{A} \mathbf{u}$, and $\widetilde{\mathbf{u}}=\mathbf{A}^{*} \mathbf{w}$.

Also if the test subspace $\mathcal{W}_{k}$ is not constructed as to approach a subspace of left eigenvectors (cf. §5.1.3) then still the use of left eigenvectors may help to improve the convergence: select $\mathbf{w}=\mathbf{W}_{k} y_{\ell}$, where $y_{\ell}$ is the left eigenvector of the projected problem.

\subsection{The basis of the search subspace and its test subspace.}

For stability reasons the basis of the search subspace as well as of the test subspace should satisfy certain orthogonality properties.

For instance, $\mathbf{v}_{k+1} \equiv \mathbf{v}^{\prime} /\left\|\mathbf{v}^{\prime}\right\|$, with $\mathbf{v}^{\prime} \equiv \mathbf{z}_{1}-\mathbf{V}_{k} \mathbf{V}_{k}^{*} \mathbf{z}_{1}$ leads to an orthonormal basis for the search subspace and $\mathbf{V}_{k}^{*} \mathbf{V}_{k}=I$. Of course, the vector $\mathbf{v}^{\prime}$ can also be computed by modified Gram-Schmidt. For accuracy reasons, this modified approach is even to be preferred.

If $\mathbf{B}$ is positive definite, then $\mathbf{v}_{k+1} \equiv \mathbf{v}^{\prime} / \gamma$ with $\mathbf{v}^{\prime} \equiv \mathbf{z}_{1}-\mathbf{V}_{k} \mathbf{V}_{k}^{*} \mathbf{B} \mathbf{z}_{1}$ and $\gamma^{2} \equiv \mathbf{v}^{\prime *} \mathbf{B} \mathbf{v}^{\prime}$, leads to a $\mathbf{B}$-orthonormal basis: $\mathbf{V}_{k}^{*} \mathbf{B} \mathbf{V}_{k}=I$ which simplifies the projected problem to $\mathbf{V}_{k}^{*} \mathbf{A V}_{k}-\vartheta I$ if $\mathcal{W}_{k}=\mathcal{V}_{k}$.

Assume that $\mathcal{W}_{k}=\mathbf{B} \mathcal{V}_{k}$ is the selected test subspace. Then $\mathbf{w}_{k+1} \equiv \mathbf{w}^{\prime} /\left\|\mathbf{w}^{\prime}\right\|$, $\mathbf{v}_{k+1} \equiv \mathbf{v}^{\prime} /\left\|\mathbf{w}^{\prime}\right\|$ with $\mathbf{w}^{\prime} \equiv \mathbf{B} \mathbf{z}_{1}-\mathbf{W}_{k} \mathbf{W}_{k}^{*} \mathbf{B} \mathbf{z}_{1}$ and $\mathbf{v}^{\prime} \equiv \mathbf{z}_{1}-\mathbf{V}_{k} \mathbf{W}_{k}^{*} \mathbf{B} \mathbf{z}_{1}$, leads to an orthonormal basis for the subspace $\mathcal{W}_{k}$ : $\mathbf{W}_{k}^{*} \mathbf{W}_{k}=I$, while $\mathbf{B V}_{k}=\mathbf{W}_{k}$. The projected problem reduces in this case to $\mathbf{W}_{k}^{*} \mathbf{A V}_{k}-\vartheta I$.

\section{The solution of the projected eigenproblem.}

The projected eigenproblem (4.2) is a relatively small eigenproblem of dimension $\operatorname{dim}\left(\operatorname{span}\left(\mathbf{V}_{k}\right)\right)$ and can be solved relatively efficiently by standard algorithms such as the QR-algorithm. Of course, if one is interested in, for instance, only the eigenvalue with largest real part, then there is no need to compute all eigenvalues of the projected problem. However, if one is interested in a interior eigenvalue, then it may be helpful to compute all Petrov values: this may facilitate the selection of the appropriate approximating eigenvalue.

The computation of the projected matrix, e.g., $\mathbf{W}_{k}^{*} \mathbf{A} \mathbf{V}_{k}$ involves the evaluation of a number of inner products. Using the fact that

$$
\mathbf{W}_{k+1}^{*} \mathbf{A V}_{k+1}=\left[\begin{array}{cc}
\mathbf{W}_{k}^{*} \mathbf{A V} & \mathbf{W}_{k}^{*} \mathbf{A} \mathbf{v}_{k+1} \\
\mathbf{w}_{k+1}^{*} \mathbf{A V} \mathbf{V}_{k} & \mathbf{w}_{k+1}^{*} \mathbf{A v}_{k+1}
\end{array}\right]
$$


if $\mathbf{V}_{k+1}=\left[\mathbf{V}_{k}, \mathbf{v}_{k+1}\right], \mathbf{W}_{k+1}=\left[\mathbf{W}_{k}, \mathbf{w}_{k+1}\right]$, reduces the number of inner products per sweep.

\section{The correction equation.}

We will have an accurate approximation for the eigenvector $\mathbf{x}$ if the angle between $\mathbf{x}$ and the search subspace $\mathcal{V}_{k}$ is sufficiently small. We can achieve small angles in two ways: by building high dimensional search subspaces $\mathcal{V}_{k}$ ( $k$ large), or by carefully selecting the vectors $\mathbf{z}_{1}$ by which we extend these subspaces $\mathcal{V}_{k}$. To make this more precise, we extend $\mathcal{V}_{k}$ by $\mathbf{z}_{1}$, where $\mathbf{z}_{1}$ is an approximate solution of

(a) the correction equation (3.7) or of

(b) the complete set of eigenvalue equations (3.5).

More accurate solutions of (3.5) will involve more computational work in terms of matrix vector multiplications for the solution of part (3.5.b), while for (3.7) the higher dimensional subspaces $\mathcal{V}_{k}$ require more vector updates in the steps where the matrices are projected onto $\mathcal{V}_{k}$ and for the construction of the Ritz vector. We have the alternative of inexpensive inner loops (where the corrections equation is solved) in combination with more expensive outer loops (where the projected eigenproblem is solved) and expensive inner loops with inexpensive outer loops. The optimal strategy will depend on the convergence properties and on costs of a matrix-vector product as compared with vector updates.

For local efficiency reasons one may prefer more accurate solutions of (3.5). However, since the quadratic convergence of the JD process will only occur for larger $k$ (the convergence is asymptotically quadratic), one may postpone the fast convergence by investing the computational effort in obtaining accurate solutions of (3.5) rather than building larger search subspaces $\mathcal{V}_{k}$. For instance, if one uses Newton's process for the solution of (3.5), then one may see a very slowly converging or even a diverging process if the initial guess (computed from results in the outer loop) is not accurate enough.

There are a number of ways to achieve efficiently more accurate solutions of (3.5) with iterative methods. One may think of

(a) preconditioning (cf. $\S 7.1$ ),

(b) using the subspace, that was constructed to solve (3.7) approximately, in order to obtain an approximate solution of (3.5),

(c) using the subspace that has been constructed in the outer loop, in order to obtain a more accurate approximation of (3.7),

(d) iterating (3.5), say, $\ell$ times $(\ell \geq 2)$ by the Jacobi correction method (as described in the paragraph following (2.3)),

(e) iterating (3.5), say, $\ell$ times with Newton process, or

(f) combinations of these strategies. 
If one uses $\ell$ steps of some iterative process to solve (3.5), as in (d) or (e), then there will be an optimal $\ell$ as far as the overall efficiency is concerned.

In the next subsections we will discuss the option of preconditioning, the other possibilities will be subject of further study.

\subsection{Preconditioning.}

If $\mathbf{M}_{p}$ is an approximation for the inverse of the projected matrix $\mathbf{F}_{p}$ in (3.8), then one may use this operator in order to get an accurate solution $\mathbf{z}$ of the correction equation (3.7) in fewer iteration steps. The preconditioner should map the image space $\mathbf{w}^{\perp}$ of $\mathbf{F}_{p}$ onto its domain space $\widetilde{\mathbf{u}}^{\perp}$. Therefore, $\mathbf{M}_{p}$ should satisfy

$$
\mathbf{M}_{p}=\left(\mathbf{I}-\frac{\mathbf{u} \widetilde{\mathbf{u}}^{*}}{\widetilde{\mathbf{u}}^{*} \mathbf{u}}\right) \mathbf{M}_{p}\left(\mathbf{I}-\frac{\widetilde{\mathbf{w}} \mathbf{w}^{*}}{\mathbf{w}^{*} \widetilde{\mathbf{w}}}\right) .
$$

Then the preconditioned correction equation for $\mathbf{z} \perp \widetilde{\mathbf{u}}$ reads as

$$
\mathbf{M}_{p} \mathbf{F}_{p} \mathbf{z}=\mathbf{M}_{p}(\mathbf{A}-\vartheta \mathbf{B})\left(\mathbf{I}-\frac{\mathbf{u} \widetilde{\mathbf{u}}^{*}}{\widetilde{\mathbf{u}}^{*} \mathbf{u}}\right) \mathbf{z}=-\mathbf{M}_{p} \mathbf{r} .
$$

Here we exploit the fact that projections are involved: applying such a map once has the same effect as applying it twice. In the context of Krylov subspace methods, the following similar observation is useful:

$$
\mathbf{M}_{p} \mathbf{F}_{p} \mathbf{y}=\mathbf{M}_{p}(\mathbf{A}-\vartheta \mathbf{B}) \mathbf{y} \quad \text { if } \quad \mathbf{y}=\mathbf{M}_{p} \tilde{\mathbf{y}},
$$

since the vectors involved in the iteration process usually can be written in the latter form.

\subsubsection{Projecting preconditioners.}

In most cases, preconditioners are specified as approximations $\mathbf{K}$ for $\mathbf{A}-\vartheta \mathbf{B}$, and such that the equation $\mathbf{K t}=\mathbf{r}$ can be solved relatively easily. For instance, Davidson [7] suggested $\mathbf{K}=\operatorname{diag}(\mathbf{A})-\vartheta \operatorname{diag}(\mathbf{B})$ (see also $[19,8]$ ). In view of our observations in $\S 3$ we expect to create better preconditioners by taking the projections into account. Therefore, we consider the inverse as a map from $\widetilde{\mathbf{u}}^{\perp}$ onto $\mathbf{w}^{\perp}$ of $\mathbf{K}_{p}$, the projection of $\mathbf{K}$,

$$
\mathbf{K}_{p} \equiv\left(\mathbf{I}-\frac{\widetilde{\mathbf{w}} \mathbf{w}^{*}}{\mathbf{w}^{*} \widetilde{\mathbf{w}}}\right) \mathbf{K}\left(\mathbf{I}-\frac{\mathbf{u} \widetilde{\mathbf{u}}^{*}}{\widetilde{\mathbf{u}}^{*} \mathbf{u}}\right) .
$$

The following propositions (Prop. 7.1, 7.2 and 7.4) express the inverse of $\mathbf{K}_{p}$ in terms of the inverse of $\mathbf{K}$. The operator $\mathbf{K}_{p}^{-1}$ denotes the inverse of $\mathbf{K}_{p}$ as a map from $\widetilde{\mathbf{u}}^{\perp}$ onto $\mathbf{w}^{\perp}$.

Proposition 7.1. If $\mathbf{K}$ maps $\widetilde{\mathbf{u}}^{\perp}$ onto $\mathbf{w}^{\perp}$ then $\mathbf{K}_{p}^{-1}$ exists with respect to the subspace $\mathbf{w}^{\perp}$, and

$$
\mathbf{K}_{p}^{-1}=\left(\mathbf{I}-\frac{\mathbf{u} \widetilde{\mathbf{u}}^{*}}{\widetilde{\mathbf{u}}^{*} \mathbf{u}}\right) \mathbf{K}^{-1}\left(\mathbf{I}-\frac{\widetilde{\mathbf{w}} \mathbf{w}^{*}}{\mathbf{w}^{*} \widetilde{\mathbf{w}}}\right)=\mathbf{K}^{-1} \text { on } \quad \mathbf{w}^{\perp} .
$$


PROOF. If $\mathbf{y} \perp \mathbf{w}$ and $\mathbf{t}$ is such that $\mathbf{K t}=\mathbf{y}$ then $\mathbf{t} \perp \tilde{\mathbf{u}}$ and $\mathbf{K}_{p} \mathbf{t}=\mathbf{y}$.

Remark 7.1. The condition in Proposition 7.1 is satisfied if, for instance, $\mathbf{K}$ is diagonal and if $\mathbf{w}=\tilde{\mathbf{u}}$ is a standard basis vector. Then, for any $\mathbf{y} \perp \mathbf{w}$, we can compute $\mathbf{K}_{p}^{-1} \mathbf{y}$ without projection: $\mathbf{K}_{p}^{-1} \mathbf{y}=\mathbf{K}^{-1} \mathbf{y}$.

In general, for non-diagonal preconditioners, the projections cannot be ignored, that is, skipping the projections may lead to slower convergence. For a numerical example, see $\S 9.3$ (see also [32]). For this general situation, it is not so obvious how to compute $\mathbf{K}_{p}$, and the following proposition may be helpful.

Proposition 7.2. If $\mathbf{K}$ is non-singular and $\tilde{\mathbf{u}}^{*} \mathbf{K}^{-1} \widetilde{\mathbf{w}} \neq 0$ then $\mathbf{K}_{p}^{-1}$ exists with respect to the subspace $\mathbf{w}^{\perp}$, and

$$
\mathbf{K}_{p}^{-1}=\mathbf{K}^{-1}\left(\mathbf{I}-\frac{\widetilde{\mathbf{w}} \mathbf{y}_{r}^{*}}{\mathbf{y}_{r}^{*} \widetilde{\mathbf{w}}}\right)=\left(\mathbf{I}-\frac{\mathbf{y}_{\ell} \tilde{\mathbf{u}}^{*}}{\widetilde{\mathbf{u}}^{*} \mathbf{y}_{\ell}}\right) \mathbf{K}^{-1} \text { on } \quad \mathbf{w}^{\perp}
$$

where $\mathbf{y}_{r}$ solves $\mathbf{K}^{*} \mathbf{y}_{r}=\widetilde{\mathbf{u}}$ and $\mathbf{y}_{\ell}$ solves $\mathbf{K}_{\ell}=\tilde{\mathbf{w}}$.

Proof. Observe that $\widetilde{\mathbf{u}}^{*} \mathbf{y}_{\ell}=\mathbf{y}_{r}^{*} \tilde{\mathbf{w}} \neq 0$. Clearly the second equality holds.

If $\mathbf{K t}=\mathbf{r}+\beta \widetilde{\mathbf{w}}$ with scalar $\beta$ such that $\widetilde{\mathbf{u}} \perp \mathbf{t}$ then $\mathbf{K}_{p} \mathbf{t}=\mathbf{r}$, leading to the first equality in (7.5).

THEOREM 7.3. If $\mathbf{K}$ is non-singular and $\widetilde{\mathbf{u}}^{*} \mathbf{K}^{-1} \tilde{\mathbf{w}} \neq 0$ then the correction equation (3.7) preconditioned by $\mathbf{K}_{p}^{-1}$ (cf. PROP. 7.2) is equivalent to

$$
\mathbf{z} \perp \tilde{\mathbf{u}} \quad \text { and }\left(\mathbf{I}-\frac{\mathbf{y}_{\ell} \widetilde{\mathbf{u}}^{*}}{\widetilde{\mathbf{u}}^{*} \mathbf{y}_{\ell}}\right) \mathbf{K}^{-1}(\mathbf{A}-\vartheta \mathbf{B})\left(\mathbf{I}-\frac{\mathbf{y}_{\ell} \widetilde{\mathbf{u}}^{*}}{\widetilde{\mathbf{u}}^{*} \mathbf{y}_{\ell}}\right) \mathbf{z}=-\mathbf{r}^{\prime}
$$

where

$$
\mathbf{y}_{\ell} \equiv \mathbf{K}^{-1} \widetilde{\mathbf{w}} \quad \text { and } \quad \mathbf{r}^{\prime} \equiv\left(\mathbf{I}-\frac{\mathbf{y}_{\ell} \widetilde{\mathbf{u}}^{*}}{\widetilde{\mathbf{u}}^{*} \mathbf{y}_{\ell}}\right) \mathbf{K}^{-1} \mathbf{r}
$$

ProOF. Since, with $\mathrm{y}_{r}$ as in Proposition 7.2,

$$
\left(\mathbf{I}-\frac{\widetilde{\mathbf{w}} \mathbf{y}_{r}^{*}}{\mathbf{y}_{r}^{*} \widetilde{\mathbf{w}}}\right)\left(\mathbf{I}-\frac{\widetilde{\mathbf{w}} \mathbf{w}^{*}}{\mathbf{w}^{*} \tilde{\mathbf{w}}}\right)=\left(\mathbf{I}-\frac{\widetilde{\mathbf{w}} \mathbf{y}_{r}^{*}}{\mathbf{y}_{r}^{*} \widetilde{\mathbf{w}}}\right)
$$

equation (7.5) shows that the preconditioned correction equation is equivalent to

$$
\mathbf{z} \perp \tilde{\mathbf{u}} \text { and }\left(\mathbf{I}-\frac{\mathbf{y}_{\ell} \widetilde{\mathbf{u}}^{*}}{\widetilde{\mathbf{u}}^{*} \mathbf{y}_{\ell}}\right) \mathbf{K}^{-1}(\mathbf{A}-\vartheta \mathbf{B})\left(\mathbf{I}-\frac{\mathbf{u} \widetilde{\mathbf{u}}^{*}}{\widetilde{\mathbf{u}}^{*} \mathbf{u}}\right) \mathbf{z}=-\mathbf{r}^{\prime}
$$

with $y_{\ell}$ and $r^{\prime}$ as in (7.7). Finally, the equivalence of (7.6) and (7.8) follows from the fact that

$$
\mathbf{z} \perp \tilde{\mathbf{u}} \Leftrightarrow \mathbf{z}=\left(\mathbf{I}-\frac{\mathbf{y}_{\ell} \widetilde{\mathbf{u}}^{*}}{\widetilde{\mathbf{u}}^{*} \mathbf{y}_{\ell}}\right) \mathbf{z} \quad \Leftrightarrow \quad \mathbf{z}=\left(\mathbf{I}-\frac{\mathbf{u} \tilde{\mathbf{u}}^{*}}{\widetilde{\mathbf{u}}^{*} \mathbf{u}}\right) \mathbf{z} .
$$


In [32] a diagonal block approximation $\mathbf{K}$ of $\mathbf{A}-\vartheta \mathbf{B}$, in combination with a block approximation of the projections using the same block structure, was reported to be successful for the application considered there.

REMARK 7.2. In the Davidson methods $[5,7,18,19]$ the search subspace is expanded by the vector $\mathbf{K}^{-1} \mathbf{r}$, the vector that appears in the first step of the computation of $\mathbf{r}^{\prime}$ (cf. TH. 7.3). In Olsen's method (cf. [21]), for symmetric eigenproblems with $\widetilde{\mathbf{u}}=\widetilde{\mathbf{w}}=\mathbf{u}$, the search subspace is expanded by the vector $\mathbf{r}^{\prime}$ (see also [29]).

If $\mathbf{K}$ is a good approximation of $\mathbf{A}-\lambda \mathbf{B}$ then $\mathbf{K}$ may be singular and the following proposition may be useful ${ }^{1}$.

PROPOSITION 7.4. If $\operatorname{Ker}(\mathbf{K})=\operatorname{span}(\mathbf{a})$ for some non-trivial vector a then there is a non-trivial vector $\mathbf{b}$ for which $\operatorname{Ker}\left(\mathbf{K}^{*}\right)=\operatorname{span}(\mathbf{b})$ and

$$
\mathbf{K}_{p}^{-1}=\left(\mathbf{I}-\frac{\mathbf{a} \tilde{\mathbf{u}}^{*}}{\widetilde{\mathbf{u}}^{*} \mathbf{a}}\right) \mathbf{K}^{-1}\left(\mathbf{I}-\frac{\tilde{\mathbf{w}} \mathbf{b}^{*}}{\mathbf{b}^{*} \tilde{\mathbf{w}}}\right) \text { on } \mathbf{w}^{\perp},
$$

where $\mathbf{K}^{-1}$ is the inverse of $\mathbf{K}$ as a map from $\mathbf{a}^{\perp}$ onto $\mathbf{b}^{\perp}$.

PROOF. If $\mathbf{K t}=\mathbf{K}(\mathbf{t}+\alpha \mathbf{a})=\mathbf{r}+\beta \tilde{\mathbf{w}}$ with scalars $\alpha$ and $\beta$ such that $\mathbf{b} \perp \mathbf{r}+\beta \tilde{\mathbf{w}}$ and $\tilde{\mathbf{u}} \perp \mathbf{t}+\alpha \mathbf{a}$ then $\mathbf{K}_{p} \mathbf{t}=\mathbf{r}$. This leads to (7.9).

\subsubsection{The augmented correction equation.}

Formulation (3.17) of the correction equation (3.7) (for the case $\mathbf{r} \perp \mathbf{w}$ ) may be more accessible for the construction of suitable preconditioners, avoiding explicit projections as in $\S 7.1 .1$.

The following lemma, for which we omit the obvious proof, is helpful for the construction inverses corresponding to equation (3.17).

LEMMA 7.5. For $\mathbf{z}$ and $\mathbf{y}$ we have

$$
\left[\begin{array}{cc}
\mathbf{I} & \mathbf{y} \\
\mathbf{z}^{*} & 0
\end{array}\right]=\left[\begin{array}{cc}
\mathbf{I} & 0 \\
\mathbf{z}^{*} & 1
\end{array}\right]\left[\begin{array}{cc}
\mathbf{I} & \mathbf{y} \\
0^{*} & -\nu
\end{array}\right] \text { where } \nu \equiv \mathbf{z}^{*} \mathbf{y}
$$

and the matrix is invertible if and only if $\mathbf{z}^{*} \mathbf{y} \neq 0$.

If $\mathbf{K}$ is an easily invertible approximation of $\mathbf{A}-\vartheta \mathbf{B}$ then the augmented equation (3.17) can be preconditioned by

$$
\left[\begin{array}{cc}
\mathbf{K} & \tilde{\mathbf{w}} \\
\tilde{\mathbf{u}}^{*} & 0
\end{array}\right]^{-1}=\left[\begin{array}{rr}
\mathbf{I} & -\mathbf{y}_{\ell} \\
0^{*} & 1
\end{array}\right]\left[\begin{array}{rr}
\mathbf{I} & 0 \\
\frac{\tilde{\mathbf{u}}^{*}}{\nu} & \frac{-1}{\nu}
\end{array}\right]\left[\begin{array}{cc}
\mathbf{K}^{-1} & 0 \\
0^{*} & 1
\end{array}\right] \text {, where }\left\{\begin{array}{l}
\mathbf{y}_{\ell} \equiv \mathbf{K}^{-1} \tilde{\mathbf{w}} \\
\nu \equiv \tilde{\mathbf{u}}^{*} \mathbf{y}_{\ell}
\end{array}\right.
$$

and

$$
\begin{aligned}
{\left[\begin{array}{cc}
\mathbf{K} & \tilde{\mathbf{w}} \\
\tilde{\mathbf{u}}^{*} & 0
\end{array}\right]^{-1}\left[\begin{array}{cc}
\mathbf{A}-\vartheta \mathbf{B} & \tilde{\mathbf{w}} \\
\tilde{\mathbf{u}}^{*} & 0
\end{array}\right] } \\
\quad=\left[\begin{array}{rr}
\mathbf{I} & -\mathbf{y}_{\ell} \\
0^{*} & 1
\end{array}\right]\left[\begin{array}{cr}
\mathbf{I} & 0 \\
\frac{\tilde{\mathbf{u}}^{*}}{\nu} & \frac{-1}{\nu}
\end{array}\right]\left[\begin{array}{cc}
\mathbf{K}^{-1}(\mathbf{A}-\vartheta \mathbf{B}) & \mathbf{y}_{\ell} \\
\tilde{\mathbf{u}}^{*} & 0
\end{array}\right] .
\end{aligned}
$$

\footnotetext{
${ }^{1}$ The idea exposed in the proposition was also suggested by Dr. F. Wubs (private communication).
} 
For more efficient computation, we prefer the factorized form at the right hand sides of equations (7.10) and (7.11).

Clearly (7.10) has also a 'left variant' (with $\mathbf{K}^{-1}$ at the left in the right hand side). The left variant may be more suitable for post preconditioning of the augmented equation (3.17). Both variants are obvious analogues of the preconditioners in (7.5); likewise, (7.11) is an analogue of (7.6).

\section{Polynomial eigenvalue equations.}

Polynomial eigenproblems are relevant for scientific modelling, for instance in mechanical systems with friction. Bai (see $\S 8$ in [2]) refers to an interesting 5 -th order problem that waits for solution. With the classical subspace methods one has to linearize the given problem, by forming the companion matrix. This matrix is not only much bigger than the order of the matrices involved in the given problem, but it is usually also very ill-conditioned. As we will see, the Jacobi-Davidson approach offers the possibility to handle the polynomial problem directly without any linearization.

For $\ell \in \mathbb{N}$, and $n \times n$ matrices $\mathbf{A}_{0}, \ldots, \mathbf{A}_{\ell}$, consider the generalized eigenproblem of finding an eigenvector $\mathbf{x}$ (non-trivial) and associated eigenvalue $\lambda \in \mathbb{C}$ such that

$$
\mathbf{A}_{0} \mathbf{x}+\lambda \mathbf{A}_{1} \mathbf{x}+\cdots+\lambda^{\ell} \mathbf{A}_{\ell} \mathbf{x}=0 .
$$

In terms of a matrix-valued polynomial $\boldsymbol{\Psi}$, we are interested in pairs $(\lambda, \mathbf{x})$ of scalars $\lambda$ and non-trivial vectors $\mathbf{x}$ for which

$$
\boldsymbol{\Psi}(\lambda) \mathbf{x}=0 \quad \text { where } \quad \boldsymbol{\Psi}(\zeta) \equiv \mathbf{A}_{0}+\zeta \mathbf{A}_{1}+\cdots+\zeta^{\ell} \mathbf{A}_{\ell} \quad(\zeta \in \mathbb{C}) .
$$

To solve this problem, we can proceed as before. Suppose we have a $k$ dimensional search subspace $\mathcal{V}_{k}$ and a $k$-dimensional projection subspace $\mathcal{W}_{k}$. Then, we can compute an approximation $\mathbf{u}$ of $\mathbf{x}$ with associated approximation $\vartheta$ of $\lambda$, by solving the projected problem:

$$
\mathbf{u} \in \mathcal{V}_{k}, \vartheta \in \mathbb{C} \text { such that } \boldsymbol{\Psi}(\vartheta) \mathbf{u} \perp \mathcal{W}_{k}
$$

We define the residual $\mathbf{r}$ by

$$
\mathrm{r} \equiv \boldsymbol{\Psi}(\vartheta) \mathrm{u}
$$

and, for a some $\tilde{\mathbf{u}}$, we correct the approximation $\mathbf{u}$ by $\mathbf{z}_{1}$, where $\mathbf{z}_{1} \perp \tilde{\mathbf{u}}$ is an approximate solution of the correction equation:

$$
\mathbf{z} \perp \tilde{\mathbf{u}} \text { and }\left(\mathbf{I}-\frac{\widetilde{\mathbf{w}} \mathbf{w}^{*}}{\mathbf{w}^{*} \widetilde{\mathbf{w}}}\right) \Psi(\vartheta)\left(\mathbf{I}-\frac{\mathbf{u} \widetilde{\mathbf{u}}^{*}}{\widetilde{\mathbf{u}}^{*} \mathbf{u}}\right) \mathbf{z}=-\mathbf{r},
$$

for relevant choices of $\mathbf{w}$ and $\tilde{\mathbf{w}}$, with $\mathbf{w}$ orthogonal to $r$. As before, the approximate correction $\mathrm{z}_{1}$ can be used to extend the search subspace $\mathcal{V}_{k}$. By choosing basis in $\mathcal{V}_{k}$ and in $\mathcal{W}_{k}$, the projected problem (8.3) is equivalent to a small eigenproblem involving a $k \times k$-matrix-valued polynomial. If the sequences of $\mathbf{u}, \mathbf{w}$ and $\tilde{\mathbf{u}}$ converge then, the choice

$$
\tilde{\mathbf{w}} \equiv \Psi^{\prime}(\vartheta) \mathbf{u}=\left(\mathbf{A}_{1}+2 \vartheta \mathbf{A}_{2}+\cdots+\ell \vartheta^{\ell-1} \mathbf{A}_{\ell}\right) \mathbf{u}
$$


leads to asymptotic quadratic convergence.

Recently, this approach with $\mathbf{w}=\widetilde{\mathbf{u}}=\mathbf{u}$ has been used successfully for the solution of quadratic eigenvalue problems associated with acoustic models [23].

\section{Numerical examples.}

The purpose of this section is to illustrate our theoretical observations with suitable experiments. We will show how various choices of subspaces and projections affect the convergence. Our examples have been chosen deliberately to be small, since that gives us the opportunity to compute the full spectrum and to compare our findings with exact results. It is not our purpose to show how well given problems can be solved with the new approach; that has been done, for a complete implementation taking care of various criteria, in [10].

Our examples have been coded in Fortran. The small projected eigenvalue problem is solved with the Householder-QR (or QZ) algorithm [13], with routines from the LAPACK library [1]. With this algorithm we computed also the complete spectra of the matrix pairs in the examples, and we refer to these results as true (or correct) eigenvalues.

First, in $\S 9.1$, we illustrate the effect of specific choices for $\tilde{\mathbf{w}}, \mathbf{w}$ and $\widetilde{\mathbf{u}}$ on the speed of convergence of JD. Then, in $\S 9.2$ we give some examples of the performance of $\mathbf{J D}$, where $\mathbf{V}$ is $\mathbf{B}$-orthogonal and $\mathbf{V}=\mathbf{W}$, comparing this version of JD applied to the generalized eigenproblem with a naive version where JD is applied to the standard eigenproblem $\mathbf{B}^{-1} \mathbf{A x}=\lambda \mathbf{x}$. In $\S 9.3$, we discuss the effect of augmenting or projecting the preconditioner. There is a tradeoff between the computational work, needed to solve the correction equation accurately enough, and the speed of convergence of JD. This issue will be discussed in $\S 9.4$. We also consider the performance of a block version (see $\S 9.5)$.

\subsection{The effect of the projections on the speed of convergence.}

In the previous sections various choices for the subspaces $\mathcal{V}_{k}$ and $\mathcal{W}_{k}$ and the vectors $\widetilde{\mathbf{w}}, \mathbf{w}$ and $\widetilde{\mathbf{u}}$ are discussed. Their implication for the speed of convergence is analyzed and it is shown theoretically that some of these choices lead to quadratic or even cubic convergence, while other choices only lead to linear or superlinear convergence. The purpose of this subsection is to illustrate by numerical examples our findings on the convergence speed.

We present some numerical results obtained for a generalized eigenvalue problem $\mathbf{A x}=\lambda \mathbf{B x}$ taken from the "Test Matrix Collection" [3], namely the Bounded Fineline Dielectric Waveguide problem of order 398. This problem stems from a finite element discretization of the Maxwell equation for propagating modes and magnetic field profiles of a rectangular waveguide filled with dielectric and $\mathrm{PEC}$ structures. The resulting matrix $\mathbf{A}$ is non-symmetric and the matrix $\mathbf{B}$ is positive definite. Both matrices are real (but may be complex in realistic applications). Of special interest are the eigenvalues with positive real part and their corresponding eigenvectors. 
The correction equations (3.7) are (approximately) solved by (at most) 10 steps of GMRES, left preconditioned with an Incomplete LU(2) factorization of $\mathbf{A}-\tau \mathbf{B}$, for $\tau=2500$. This had the effect that the correction equations were solved with a relative residual norm reduction of $10^{-9}$ as soon as the relative residual norm of the selected Petrov pair became smaller than $10^{-2}$. We choose the starting vectors $\mathbf{v}=\mathbf{v}_{0}$ and $\mathbf{w}=\mathbf{w}_{0}$ for the first sweep both equal to the vector with all ones scaled to unit length.

The selected Petrov pair, was the one with the Petrov value closest to $\tau$ and the iterations were stopped as soon as the relative residual norm for the Petrov vector was smaller than $10^{-9}$.

Since the 5 rightmost eigenvalues of this problem are (to 5 digits of accuracy):

$$
\begin{aligned}
& \lambda_{1}=+2.9483 e 03 \\
& \lambda_{2}=+4.0338 e 02, \\
& \lambda_{3}=-1.0082 e 03 \\
& \lambda_{4}=-1.5907 e 03 \\
& \lambda_{5}=-2.0820 e 03
\end{aligned}
$$

our choice of $\tau$ leads to convergence of the Petrov pair to the largest eigenvalue $\lambda_{1}$.

\subsubsection{Linear versus quadratic.}

We start with considering the following choices for $\widetilde{\mathbf{w}}, \mathbf{w}$ and $\widetilde{\mathbf{u}}$ :

1. $\widetilde{\mathbf{w}}=\mathbf{w}=\widetilde{\mathbf{u}}=\mathbf{u}$, expecting linear convergence (cf. Theorem 3.4),

2. $\widetilde{\mathbf{w}}=\mathbf{B u}, \widetilde{\mathbf{u}}=\mathbf{w}=\mathbf{u}$, expecting quadratic convergence (cf. $\S 3.1$ ), and

3. $\widetilde{\mathbf{w}}=\mathbf{B u}, \widetilde{\mathbf{u}}=\mathbf{B}^{*} \mathbf{u}, \mathbf{w}=\mathbf{u}$, also expecting quadratic convergence (cf. §3.3).

Figure 9.1 shows the convergence of JD for these choices when we restart at every iteration (taking $\mathbf{v}_{0}=\mathbf{w}_{0}=\mathbf{u}$ ) and Figure 9.2 shows the convergence for full $\mathbf{J D}$ with $\mathbf{W}=\mathbf{V}$. In these figures, $\log _{10}\|\mathbf{r}\| / c$ is plotted, where $\mathbf{r}$ is the residual of the Petrov pair and $c$ is the norm of the initial residual. As can be seen clearly from Figure 9.1, the speed of convergence confirms our expectations. Taking the proper projections has a dramatic influence on the speed of convergence, changing linear into quadratic behavior. Notice that there is hardly any difference in the speed of convergence for the last two cases.

By comparing Figure 9.1 with Figure 9.2 we can see the benefits of an increasing search and test subspace: it has an accelerating effect, changing linear into superlinear convergence and even the quadratic convergence is faster (the cases 2 and 3 coincide in the figure). In fact, there is not much difference between the three choices.

REMARK 9.1. From the point of view of overall performance and efficiency, we recommend the second choice. The additional matrix multiplication (compared with the first choice) with $\mathbf{B}$ can be compensated by combining its computation with the computation of the residual but this cannot be done for case 3 where the transposed matrix $\mathbf{B}^{*}$ is involved. 


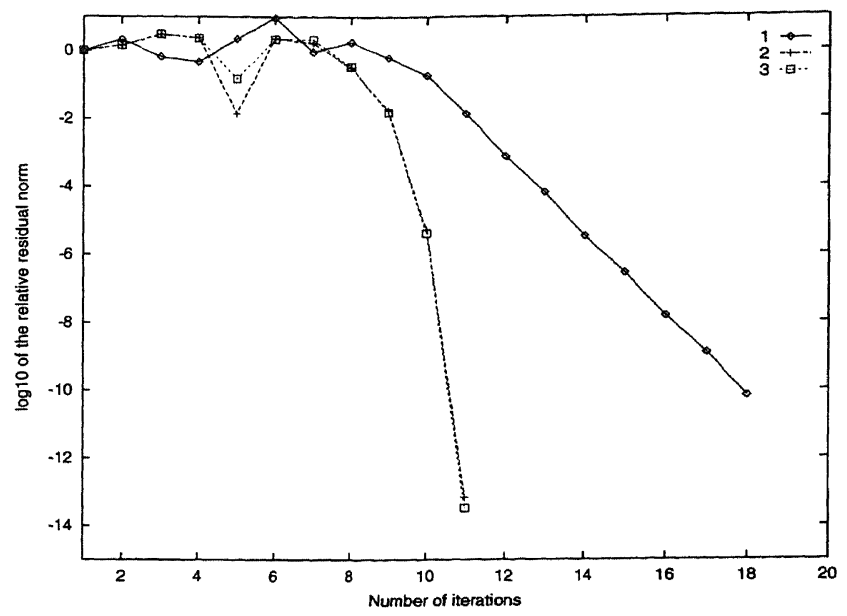

Figure 9.1: Example §9.1. Restarted JD.

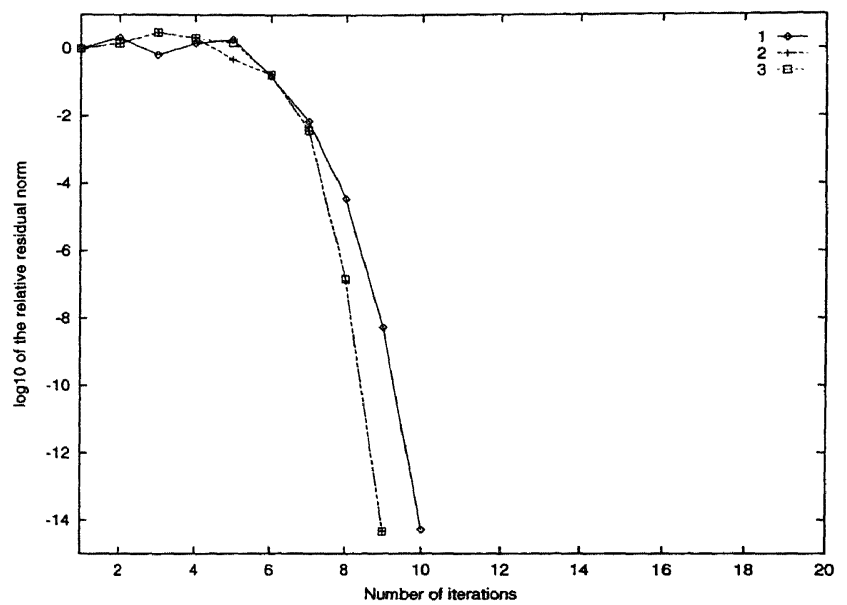

Figure 9.2: Example §9.1. Full JD.

\subsubsection{Quadratic versus cubic convergence}

In $\S 3.2$ the convergence with left eigenvectors is discussed. The statements made in that section about the speed of convergence are nicely confirmed by our numerical experiments, if we make the following choices for $\widetilde{\mathbf{w}}, \mathbf{w}$ and $\widetilde{\mathbf{u}}$ :

1. $\widetilde{\mathbf{w}}=\widetilde{\mathbf{u}}=\mathbf{u}, \mathbf{w}=\mathbf{z}$, where $\mathbf{z}$ is the left Petrov vector. This should lead to faster than linear convergence (cf. Theorem 3.4);

2. $\widetilde{\mathbf{w}}=\widetilde{\mathbf{u}}=\mathbf{u}, \mathbf{w}=\mathbf{z}$, where $\mathbf{z}$ is the approximation for the left eigenvector obtained by solving the adjoint correction equation. This should lead to quadratic convergence (cf. Remark 3.5); 
3. $\widetilde{\mathbf{w}}=\mathbf{B u}, \widetilde{\mathbf{u}}=\mathbf{B}^{*} \mathbf{z}, \mathbf{w}=\mathbf{z}$, where $\mathbf{z}$ is as above. Now we may expect cubic convergence, according to the last part of Remark 3.5.

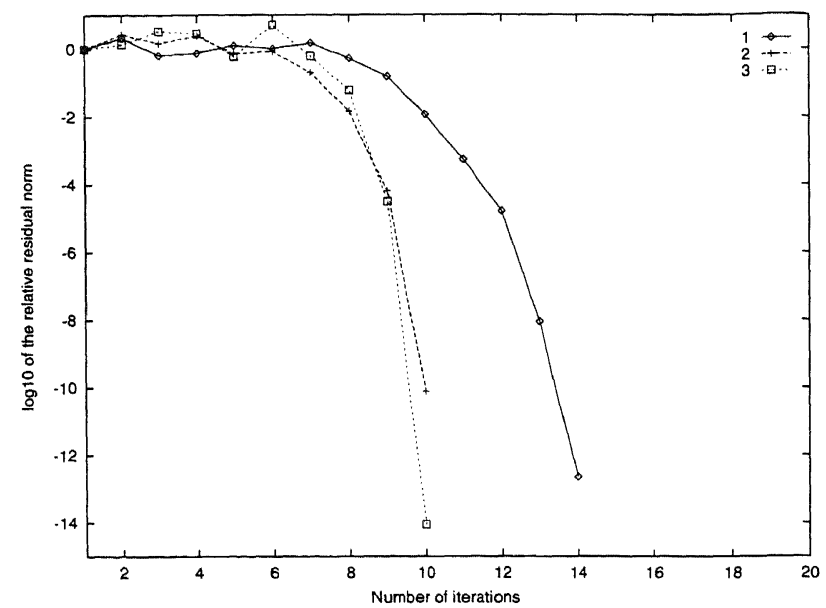

Figure 9.3: Example §9.1. Restarted JD using left Petrov vectors.

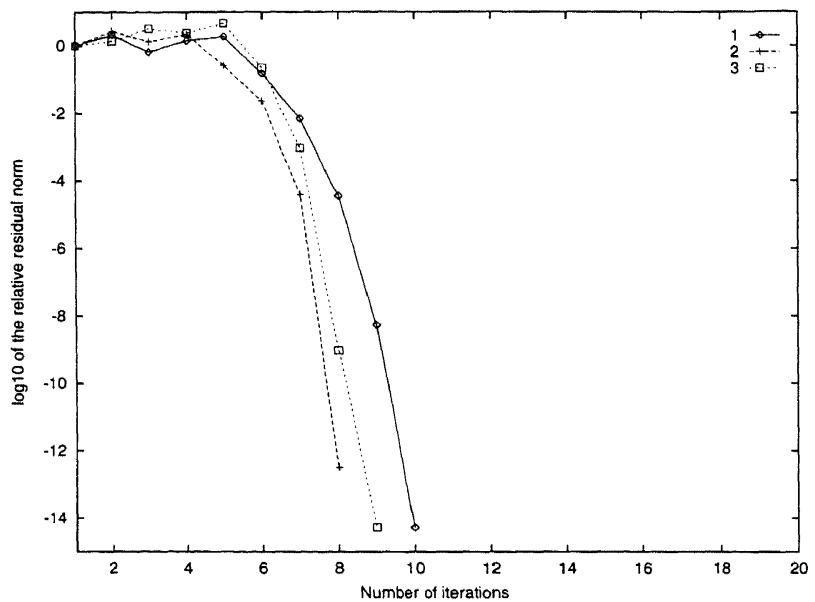

Figure 9.4: Example §9.1. Full JD using left Petrov vectors.

Figure 9.3 shows the convergence of JD for these choices when we restart at every iteration (with $\mathbf{v}_{0}=\mathbf{u}$ and $\mathbf{w}_{0}=\mathbf{z}$ ), and Figure 9.4 shows the convergence for full JD where now the test subspace is spanned by the approximations $\mathbf{z}$ of left eigenvector. Again, the speed of convergence is in line with our expectations. Using the left Petrov vector (cf. §5.2) speeds up the convergence, which is now superlinear. Solving the adjoint correction equation and using the approximation to the left eigenvector results in quadratic, or even cubic speed of convergence 
(note the reduction of the relative residual norm from $10^{-5}$ to $10^{-15}$ in the final step), when the proper projections are used. And in addition, for the last two choices, one obtains the left eigenvector almost for free.

Figure 9.4 shows the accelerating effect of increasing search and test subspace.

REMARK 9.2. Using the left Petrov vector speeds up the convergence, but there is a catch: it may happen that this left Petrov vector does not converge and this may result in slow or even no convergence for the JD process.

REMARK 9.3. Incorporating (approximations to) the left eigenvector in JD improves the speed of convergence, but at the cost of solving the adjoint correction equation (cf. (3.15)). This almost doubles the computational work, which is in general not compensated through a reduction in the number of iterations. Therefore, it appears that such a scheme can only be efficient when used for applications where both left and right eigenvectors are desired.

\subsection{Reducing a generalized eigenproblem to a standard one.}

Now we consider an example of order 80 . The nonsymmetric matrix $\mathbf{A}$ is tridiagonal and has the following non-zero entries (all other entries are zero):

$$
a_{i j}=\left\{\begin{aligned}
i & \text { if } j=i \\
1 & \text { if } j=i+1 \\
-1 & \text { if } j=i-1
\end{aligned}\right.
$$

and the symmetric matrix $\mathbf{B}$ is pentadiagonal, with the following non-zero entries:

$$
b_{i j}=\left\{\begin{aligned}
2 & \text { if } j=i \\
-1 & \text { if } j=i+1 \\
-1 & \text { if } j=i-1 \\
1 & \text { if } i=1 \text { and } j=n \\
1 & \text { if } i=n \text { and } j=1
\end{aligned}\right.
$$

Note that $\mathbf{B}$ is positive-definite. This allows for the construction of a $\mathbf{B}$-orthonormal basis, i.e., $\mathbf{V}^{*} \mathbf{B V}=I$. We selected $\mathbf{W}=\mathbf{V}$, and then the projected generalized eigenproblem reduces to a standard one. If $\mathbf{z}$ is the approximate solution of the correction equation, the set $\left[\mathbf{V}_{k}, \mathbf{z}\right]$ is $\mathbf{B}$-orthonormalized with results in $\mathbf{V}_{k+1}$, via the modified Gram-Schmidt $\left(\operatorname{Mod} G S_{B}\right)$ procedure (see e.g. $\left.[13,24]\right)$ :

$$
\text { for } \begin{aligned}
i & =1, \ldots, k \text { do: } \\
\alpha & \equiv \mathbf{v}_{i}^{*} \mathbf{B z} \\
\mathbf{z} & \equiv \mathbf{z}-\alpha \mathbf{v}_{i}
\end{aligned}
$$

end;

$\beta \equiv\|\mathbf{z}\|_{B}$

$\mathbf{v}_{k+1} \equiv \mathbf{z} / \beta$.

Here we introduced the $\mathbf{B}$-norm of a vector: $\|\mathbf{z}\|_{B}=\left(\mathbf{z}^{*} \mathbf{B} \mathbf{z}\right)^{\frac{1}{2}}$. For an efficient implementation of this $\mathbf{B}$-orthogonalization process it is convenient to store the matrix $\mathbf{B V}$ in addition to the matrix $\mathbf{A V}$, which is necessary for the computation of the projected matrix $\mathbf{V}^{*} \mathbf{A V}$. 
Table 9.1: Example §9.2. Number of outer iterations for different inner iteration processes.

\begin{tabular}{|c|c|c|c|c|}
\hline GMRES & \multicolumn{2}{|c|}{ JD Ref. [4] } & \multicolumn{2}{c|}{ JD ALG. 4.2 } \\
\hline$m$ & \# iter & \# MVs & \# iter & \# MVs \\
\hline 5 & 3490 & 62805 & 91 & 1082 \\
10 & 151 & 4953 & 29 & 618 \\
15 & 46 & 2163 & 20 & 610 \\
20 & 29 & 1767 & 17 & 674 \\
25 & 20 & 1485 & 12 & 574 \\
30 & 20 & 1770 & 11 & 622 \\
\hline
\end{tabular}

The in absolute value largest eigenvalue of this matrix pair is real and its value is

$$
\lambda=34865.927904249 \ldots
$$

This eigenvalue is well separated from the other eigenvalues. We have tried to obtain an approximation for this eigenvalue with our JD method (ALG. 4.2). The starting vector $\mathbf{v}_{1}$ is chosen as $\alpha(1, \ldots, 1)^{T}$, with $\alpha$ such that the $\mathbf{B}$-norm of $\mathbf{v}_{1}$ equals 1 . The correction equation is solved approximately with $m$ steps of GMRES with initial guess 0 and without preconditioning.

According to (3.16), the correction equation is embedded in the entire $n$-space with $\mathbf{w}=\mathbf{u}$ and $\widetilde{\mathbf{w}}=\widetilde{\mathbf{u}}=\mathbf{B u}$ (recall that $\mathbf{B}$ is symmetric). The algorithm is restarted every 10 iterations with the current eigenvector approximation, i.e., when $k=\operatorname{dim}(\operatorname{span}(\mathbf{V}))=10$ in Algorithm 4.2 we set $k=\ell=1$ and $\mathbf{v}_{1}=\mathbf{u}$. The eigenvector approximations $\mathbf{u}$ were normalized to have $\mathbf{B}$-norm 1 and the algorithm was stopped if the residual norm is smaller than $10^{-8}$.

In Table 9.1 we have listed the number of outer iterations for convergence for different numbers of GMRES steps: from 5 up to 30 . We compare the results of this $\mathbf{J D}$ variant with the variant for which $\widetilde{\mathbf{w}}=\mathbf{B u}, \mathbf{w}=\mathbf{B}^{-*} \mathbf{u}, \widetilde{\mathbf{u}}=\mathbf{u}$. The latter approach corresponds to $\mathbf{J D}$ with $\widetilde{\mathbf{u}}=\mathbf{w}=\widetilde{\mathbf{w}}=\mathbf{u}$ applied to the standard eigenproblem $\mathbf{B}^{-1} \mathbf{A} \mathbf{x}=\lambda \mathbf{x}$, and has been discussed in detail in [4]. Note that the second variant requires inversion of $\mathbf{B}^{*}$ (or exact solution of a system involving $\mathbf{B}^{*}$ ). In order to have a fair comparison we terminate this process as soon as the eigenvalue approximation has reached the same accuracy as obtained with the first, inversion-free, variant. From Table 9.1 we see that convergence is much faster for the first approach. Moreover, the work per iteration for the second variant is $50 \%$ more. This is reflected in the total number of matrixvector multiplications (MVs), also given in the table, when we take the numbers of GMRES iterations into account.

When we increase the number of GMRES steps, then the convergence is improved for this example. This is not necessarily the case, as will be shown by the example in $\S 9.3$. For 30 GMRES steps convergence is reached in only 11 outer iterations. The convergence history for this inversion-free process is presented in Table 9.2. In the second column we have listed the eigenvalue approximations. 
Table 9.2: Example §9.2. Convergence history of the inversion-free JD method with 30 GMRES steps.

\begin{tabular}{|c|r|r|r|}
\hline iteration & $\begin{array}{c}\text { eigenvalue } \\
\text { approximation }\end{array}$ & $\begin{array}{c}\text { residual } \\
\text { norm }\end{array}$ & $\begin{array}{c}\text { residual } \\
\text { norm GMRES }\end{array}$ \\
\hline 1 & 810.000000000 & 1135.79 & $2.469 \mathrm{E}-2$ \\
2 & 13206.799065923 & 4053.61 & $2.728 \mathrm{E}-1$ \\
3 & 13274.221312254 & 3812.23 & $2.913 \mathrm{E}-1$ \\
4 & 32278.051603129 & 1897.00 & $1.005 \mathrm{E}-1$ \\
5 & 33629.919920062 & 636.30 & $2.331 \mathrm{E}-1$ \\
6 & 34809.830442647 & 489.94 & $2.791 \mathrm{E}-2$ \\
7 & 34865.920209700 & 12.12 & $2.473 \mathrm{E}-3$ \\
8 & 34865.927902280 & $2.676 \mathrm{E}-2$ & $2.227 \mathrm{E}-2$ \\
9 & 34865.927904258 & $1.113 \mathrm{E}-3$ & $3.824 \mathrm{E}-3$ \\
10 & 34865.927904250 & $2.503 \mathrm{E}-6$ & $3.199 \mathrm{E}-3$ \\
11 & 34865.927904249 & $8.265 \mathrm{E}-9$ & \\
\hline
\end{tabular}

Note that the true eigenvalue is reproduced up to machine precision in the final iteration. In the third column the residual norm of the eigenpair is shown. We observe that the convergence is at least more than linear, although the correction equations are solved with a very modest accuracy, as can be seen from the last column.

\subsection{How to include a preconditioner?}

Our next example is from a magnetohydrodynamics (MHD) model, where the interaction of hot plasmas and a magnetic field is studied. These MHD phenomena occur, e.g., in the solar corona and in thermonuclear fusion reactors. The dynamical behavior of magnetically confined plasmas is described by the MHD equations, which form a system of coupled nonlinear PDEs. The stability analysis of the linearized MHD equations leads to a generalized non-Hermitian eigenproblem. For more details on the physical background, see for instance [12].

The matrix $\mathbf{A}$ in this MHD-eigenvalue problem is non-Hermitian and the matrix $\mathbf{B}$ is positive-definite Hermitian. Both matrices are block tridiagonal with rather dense blocks. Quite large MHD eigenproblems have been successfully solved with a generalized nonsymmetric Lanczos procedure [6] and with an implicitly restarted Arnoldi method [16]. The latter method was a modification of a method proposed by Sorensen [31]. In both methods a Shift-and-Invert strategy is used, which requires the LU factorization of the shifted matrix $\mathbf{A}-\mu \mathbf{B}$. The additional storage requirements due to the $L$ and $U$ factors put severe limitations on the maximum problem size that can be handled with these methods on a given computer. In the Jacobi-Davidson algorithm the factorization of either matrix is avoided, which may in principle enable plasma physicists to study larger matrix problems. Several other algorithms that avoid factorization of a matrix have been presented in the literature, see e.g. [27, 28], but to our knowl- 
edge they only apply well to the symmetric positive definite case, i.e. $\mathbf{A}, \mathbf{B}$ symmetric and $\mathbf{B}$ positive definite.

What makes this problem extremely difficult is that the eigenvalues of interest are in the interior of the spectrum, see Figure 9.5. In fact, there are many large eigenvalues, and almost any approach that avoids inversion, like for instance the standard Arnoldi method, tends to find these large dominating eigenvalues. In this section we will study a very small MHD test problem, in order to see whether an inverse free Jacobi-Davidson iteration method is feasible at all, and also in order to get an impression of how well the Jacobi-Davidson correction equation has to be solved.

The MHD test problem, that we will solve with the Jacobi-Davidson method, is obtained from [16]. The order of the matrices is $n=416$, the size of the blocks is 16 by 16 . The interesting part of the spectrum in MHD problems is determined by an interior branch of eigenvalues, known as the Alfvén spectrum. The relevant part of the spectrum, which includes the Alfvén branch, is shown in the lower figure of Figure 9.5. Note the strong clustering of unwanted eigenvalues around the origin; other much larger eigenvalues are shown in the upper part of Figure 9.5.

In order to force convergence towards interior eigenvalues, we select the Petrov values $\vartheta$ that are closest to some target value $\tau$, i.e. we search for the eigenvalue closest to $\tau$. For $\tau$ we take $\tau=-0.3+0.65 \mathrm{i}$, indicated by the square box ( $\square$ ) in Figure 9.5. As in $\S 9.2$ we construct a B-orthonormal basis, starting with $\mathbf{v}_{1}=\alpha(1, \ldots, 1)^{T}$, with $\alpha$ such that the $\left\|\mathbf{v}_{1}\right\|_{B}=1$.

We compare the results for two different forms for the correction equation:

(a) the embedded correction equation (3.16)

(b) and the augmented correction equation (3.17),

and we take $\mathbf{w}=\mathbf{u}$ and $\tilde{\mathbf{w}}=\widetilde{\mathbf{u}}=\mathbf{B u}$ for both situations.

It turns out that without preconditioning the correction equation was not solved sufficiently accurately to achieve convergence to the desired eigenvalue.

For the augmented correction equation we follow the prescription of (7.10) and (7.11), where we explicitly compute $\mathbf{y}$ and $\nu$. For $\mathbf{K}$ we take the block diagonal part of $\mathbf{A}-\tau \mathbf{B}$, with the blocks $2 \times 2$ block matrices (i.e., the blocks in $\mathbf{K}$ have size $32 \times 32$ ). Note that $\mathbf{K}$ is computed only once for the initial value of $\tau$.

We precondition the embedded correction equation with $\mathbf{K}$ without any modification. We use the same $\mathbf{K}$ as for the augmented correction equation. In contrast to the augmented correction equation (3.17), the embedded equation (3.16) is formulated in the whole space and it is an option to apply $\mathbf{K}$ without any modification. However, we will show that this is not an advisable option. In exact arithmetic, the augmented formulation is equivalent to the projected one. This seems to be the case also in finite precision arithmetic. Therefore, if the formulation using projections is preferred, we advise to project the preconditioner as well. 

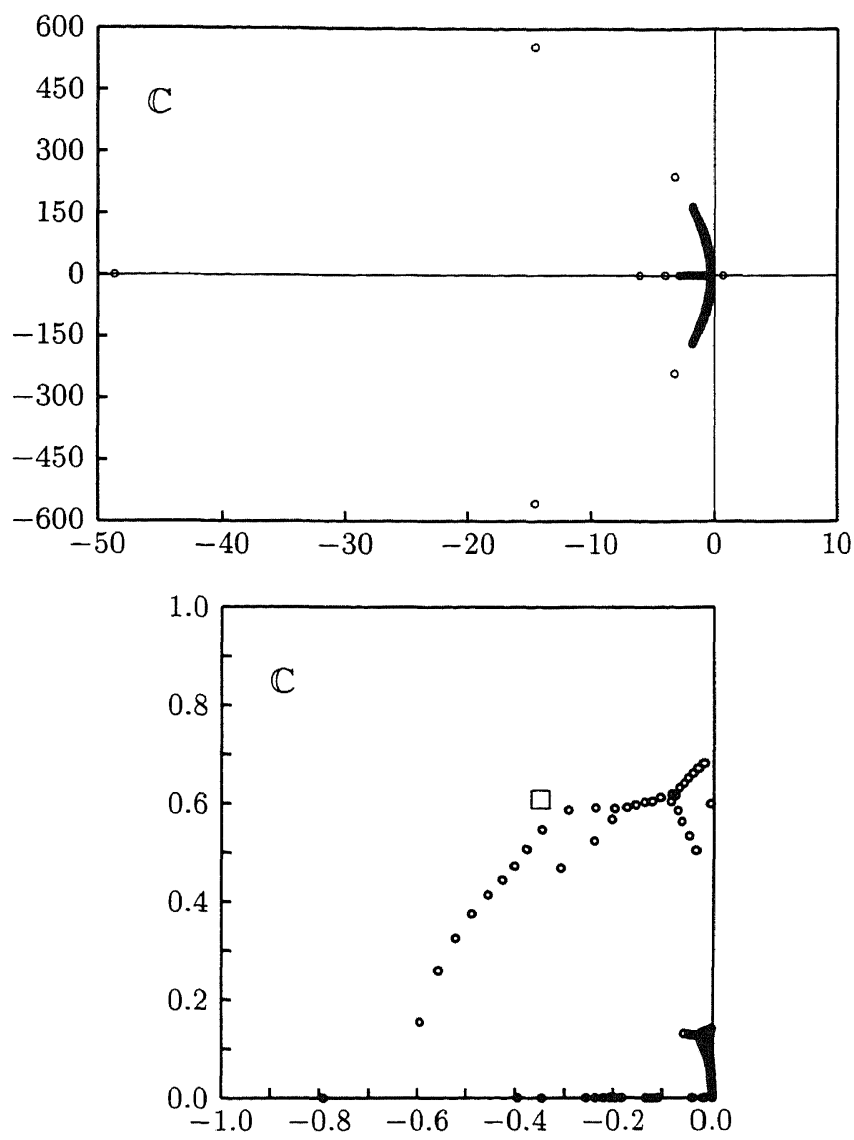

Figure 9.5: The spectrum of the MHD test problem (cf. §9.3).

The top figure gives an overview of the complete spectrum, while the bottom figure shows an amplification of the relevant part. Note that the figures have different scalings. Correct eigenvalues are shown (o) and the location of the target ( $\square$ ).

The correction equations are solved approximately with $m$ steps of full GMRES with initial guess 0 . The maximum dimension of the subspaces $\mathcal{W}_{k}$ and $\mathcal{V}_{k}$, that are constructed in the iteration process, is 50 and we restart with the current eigenvector approximation. Eigenvector approximations are normalized to have B-norm 1 and the algorithm is terminated when the residual norm of the approximate eigenpair is smaller than $10^{-8}$.

In Table 9.3 we present the total number of outer iterations necessary to achieve convergence to the desired eigenvalue. We do this for the two different inner iteration processes, where we either solve the embedded correction equa- 
Table 9.3: Example §9.3. Number of outer iterations for different inner iteration processes.

\begin{tabular}{|c|c|c|}
\hline GMRES & $\begin{array}{c}\text { Embedded } \\
\text { Correction Equation }\end{array}$ & $\begin{array}{c}\text { Augmented } \\
\text { Correction Equation }\end{array}$ \\
\hline$m$ & \# J-D iterations & \# J-D iterations \\
\hline 15 & no conv & 197 \\
20 & 133 & 48 \\
25 & 84 & 77 \\
30 & 75 & 40 \\
35 & 47 & 34 \\
40 & 41 & 33 \\
45 & 41 & 29 \\
50 & 34 & 42 \\
55 & 31 & 27 \\
60 & 35 & 29 \\
65 & 29 & 27 \\
70 & 26 & 22 \\
75 & 37 & 25 \\
80 & 24 & 24 \\
85 & 24 & 24 \\
90 & 29 & 25 \\
95 & 36 & 19 \\
100 & 21 & 20 \\
105 & 17 & 18 \\
110 & 16 & 17 \\
115 & 13 & 21 \\
120 & 11 & 16 \\
\hline
\end{tabular}

tion or the augmented correction equation, each approximately with $m$ steps of GMRES. We vary the number of GMRES steps $m$ from 15 up to 120 .

From Table 9.3 we observe that convergence is already achieved when we approximate the solution of the augmented correction equation with only 15 GMRES steps. Convergence is in general obtained in fewer iterations for the augmented correction equation with an augmented preconditioner than for the embedded correction equation with unmodified preconditioner (except for large $m(m \geq 80)$ : then the results are comparable).

Note that simply increasing the number of GMRES steps $m$, that is, selecting a better preconditioner, will not a priori lead to better convergence results. For instance, for $m=45$ we need 29 outer iterations for convergence with the augmented correction equation, whereas for $m=50$ the number of outer iterations is 42. An explanation for this is suggested by the path that the approximate eigenvalues follow to the desired eigenvalue. It turns out that for $m=50$ the convergence is much more affected by nearby other eigenvalues than for $m=45$.

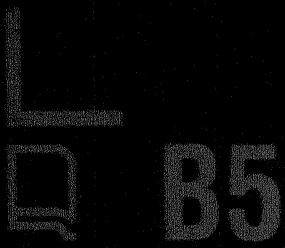




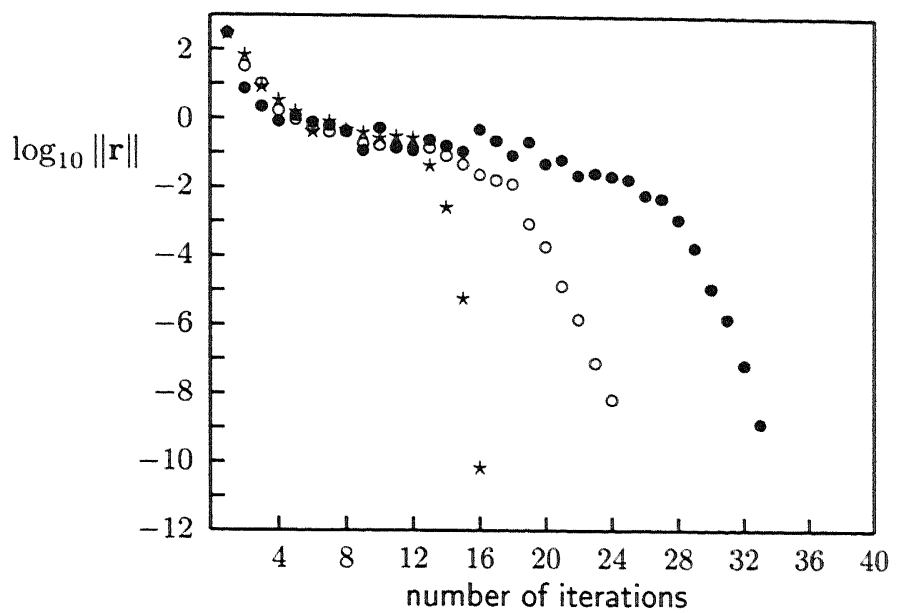

Figure 9.6: Example $\S 9.3$. Convergence history MHD problem $(n=416)$ : norm of the residual vector $\mathbf{r}$. The augmented correction equation is solved approximately with 120 GMRES iteration steps $(\star)$ ), 80 GMRES iteration steps $(0)$ and 40 GMRES iteration steps $(\bullet)$.

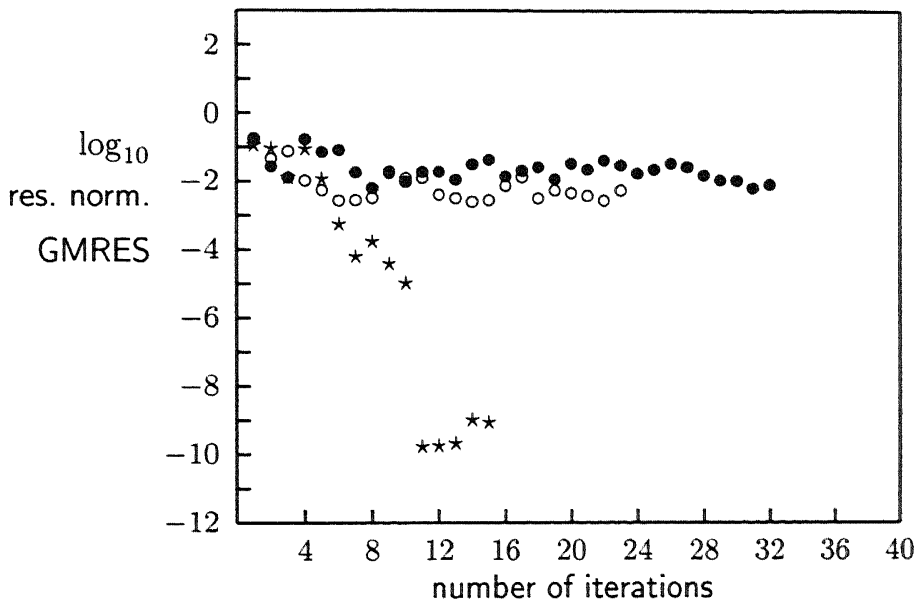

Figure 9.7: Example §9.3. Residual norm for the approximate solution of the augmented correction equation obtained with $m$ steps of the GMRES algorithm: $m=120$ $(\star), m=80(\circ)$ and $m=40(\bullet)$.

In Figure 9.6 we have plotted the $\log _{10}$ of the residual norm of the eigenpair approximation as a function of the outer iteration number. The augmented correction equation is solved approximately with 120 GMRES steps ( $\star$ ), 80 GMRES steps (०) and 40 GMRES steps $(\bullet)$. The residual norm of the approximate solution of the augmented correction equation is displayed in Figure 9.7. 
In Figure 9.6 we observe that the convergence is asymptotically quadratic if the correction equation is solved with high accuracy (120 GMRES steps). This mimics the situation where we do exact inverse. If we decrease the level of accuracy for the solution of the correction equation, that is, if we use only 80 or 40 GMRES steps, then the convergence has a more linear behavior. An explanation may be deduced from Figure 9.7, where we see that with 120 steps of GMRES the linear systems are solved very accurately during the final iterations, whereas for 80 or 40 steps only a modest accuracy is achieved for the approximate solution of the correction equation, even during the final iterations. The results for 120 GMRES steps in Figure 9.7 indicate that the condition number of the correction equation improves during the convergence process, as might have been anticipated, since the eigenpair approximation moves towards the eigenpair. Figures 9.6 and 9.7 also illustrate that the progress made during the initial iterations is virtually the same, whether we solve the correction equation rather inaccurately (with 40) or to high accuracy (120 GMRES steps); this indicates an relatively early stagnation in the GMRES inner iteration process. Therefore it is probably more economical to solve the correction equation during the initial outer iterations with only a limited number of inner iteration steps and to increase this number as the convergence proceeds; see also $\S 9.4$

In this example we have seen that it is in principle possible to use the JacobiDavidson method for interior eigenvalues without the necessity for exact inversion of any matrix. The total number of matrix evaluations for this small example makes an iterative method completely unattractive with respect to a direct method. However, if we are in the situation that we cannot invert any of the involved matrices, and if the distribution of the eigenvalues for the given problem is more or less the same as for our small test problem, then we see that is is not necessary to solve the correction equation very accurately. For the test problem the 11 Jacobi-Davidson steps with the accurate solver (120 steps with GMRES) results in 3632 matrix vector operations, whereas the 75 Jacobi-Davidson steps with the more inaccurate solver (20 steps of GMRES) requires 'only' 1976 iteration steps. Unfortunately, it is impossible to predict the proper choice for the number of GMRES steps. This example really tells us that in practice we will need rather powerful preconditioners for the correction equation.

\subsection{The computational costs.}

In view of our theoretical results in $\S 3.1$, we expect a faster converging JD process if the correction equation is solved more accurately. The numerical results in the previous subsections are in line with these observations: the number of outer iterations tends to decrease if the number of inner iterations increases. Unfortunately, a more accurate solution of the correction equation will involve more computational costs. The example in this section will illustrate that these costs can be compensated for by the faster convergence of the outer iteration.

For problems of interest, the matrices will be sparse, and a few iteration steps may not be expected to yield an accurate eigenpair approximation. Therefore, 
the vector updates and inner products, both in the outer iteration as well as in the inner iteration, will form a substantial part of total computational effort: to get an impression of the total amount of work to compute an eigenpair it is not enough to count the number of MVs.

Our example from the previous subsection does not give a realistic impression of the trade-offs in the computational costs: this model example is very small and a feasible preconditioner for large realistic MHD examples is still not available.

As in $\$ 9.1$ we take a Dielectric Waveguide problem from the "Test Matrix Collection" [3]. Except for the order, which is now 782, the present problem has the same characteristics as the smaller one in $\S 9.1$. Again we search for the eigenvalue $\lambda$ with largest real part $(\lambda \approx 2523)$ by selecting the Petrov value with largest real part. We precondition with the ILU(0) of $\mathbf{A}-\tau \mathbf{B}$, with $\tau=2500$.

The eigenvalue with largest real part is almost double, which makes this example more difficult than the one in $\$ 9.1$. The 5 rightmost eigenvalues (computed with JD) are (in 5 decimal places):

$$
\begin{aligned}
& \lambda_{1}=2.5233 e 03-i 3.1674 e-05 \\
& \lambda_{2}=2.4843 e 03+i 1.0288 e-04 \\
& \lambda_{3}=1.2640 e 03-i 2.1413 e-04 \\
& \lambda_{4}=5.6467 e 02-i 1.3443 e-05 \\
& \lambda_{5}=-1.1373 e 03-i 2.7123 e-04
\end{aligned}
$$

We select $\mathbf{w}=\widetilde{\mathbf{w}}=\mathbf{B u}$ and $\widetilde{\mathbf{u}}=\mathbf{u}$. Observe that the preconditioner has to map the image space in the correction equation to the domain space (cf. $\S 3.3$ and 7.1). We incorporate the preconditioner as explained in THEOREM 7.3. Our test subspace is spanned by $\mathbf{B V}$.

As an initial guess for $\mathbf{v}$ and $\mathbf{w}$ we take the normalized vector with all coordinates equal. In all runs, in the first $10 \mathrm{JD}$ sweeps, we solve the correction equation with 1 step of preconditioned GMRES; in the subsequential sweeps we allow more GMRES steps. Such an approach was already suggested at the end of $\S 9.3$. This may be motivated by the fact that we find the exact eigenvector in the next step if we solve the correction equation exactly with $\lambda$ instead of $\vartheta$ : actually we replaced $\lambda$ by $\vartheta$ arguing that $\vartheta$ is the best approximation of $\lambda$ that is available (cf. $\S 2$ ). However, especially in the initial stage of the process, $\vartheta$ may be worse than, say, $\tau$. Since the present preconditioner is designed for a correction equation with $\tau$ rather $\vartheta$, the result of 1 step of preconditioned GMRES applied to the " $\vartheta$-correction equation" will actually approximate the solution of the " $\tau$-correction equation" better, whereas the result of more steps of GMRES is a better approximation for the solution of the " $\vartheta$-correction equation".

The results are shown in the Figures 9.8-9.10, where, for several values of $m$, the correction equation is solved with (at most) $m$ steps of GMRES. Figure 9.8 gives the number of JD sweeps required to obtain an eigenpair approximation with a residual norm less than $10^{-9}$. As may be expected this number decreases if $m$ increases. For the number of MVs, shown in Figure 9.9, we have the opposite situation. In terms of computational work, there is not such a monotonic 


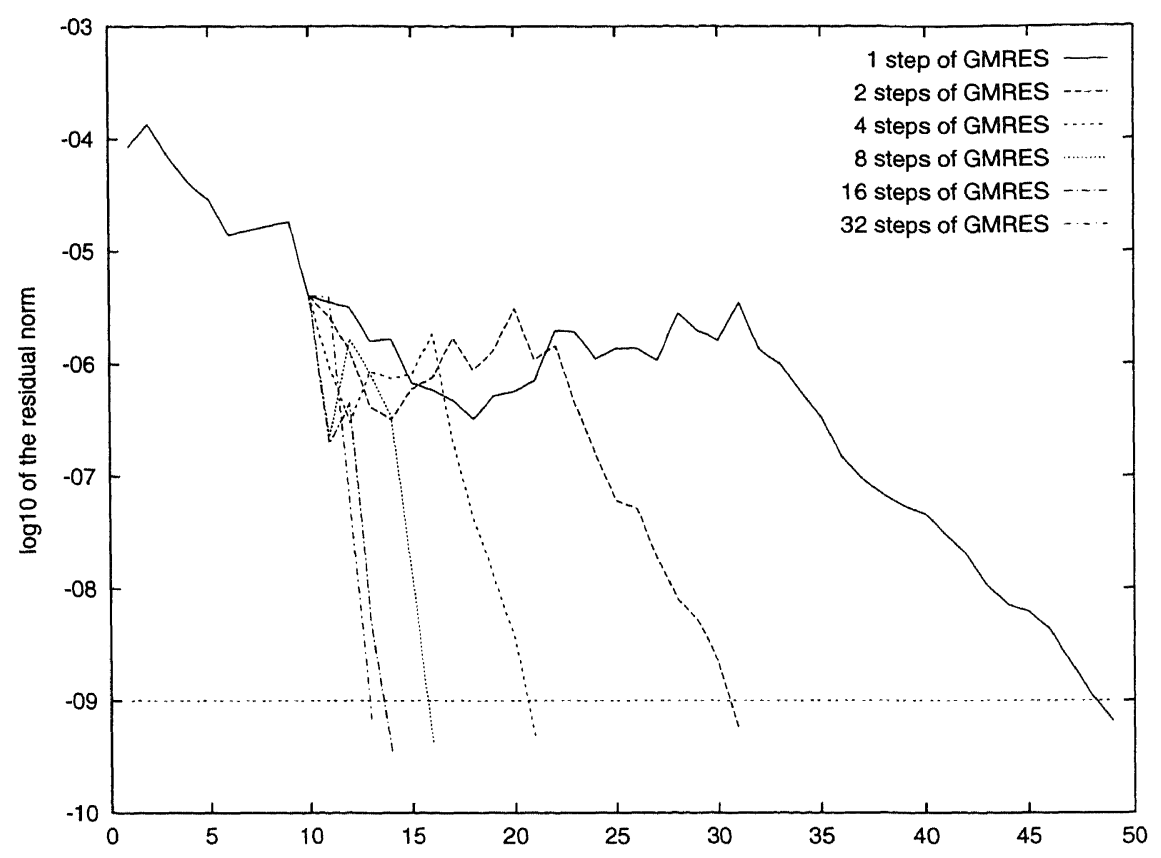

Figure 9.8: Example §9.4. Number of JD iterations.

dependence on $m$. In Figure 9.10 we give the number of floating point operations (in millions) (neglecting the operations at low dimensional levels). The method turns out to be four times faster with $m=8$ than with $m=1$.

Arnoldi did not converge within $80 \times 10^{6}$ flops. In other examples, we did not obtain convergence with a few steps of GMRES, while in other examples 1 step of GMRES turned out to be the most efficient approach. What the best strategy is depends on the problem and, of course, on the quality of the preconditioner.

\subsection{Computing several eigenvalues simultaneously.}

So far we have restricted our numerical tests to the computation of a single eigenvalue. In order to obtain several eigenvalues at the same time, we try a block variant of the basic Jacobi-Davidson algorithm (ALG. 4.2), that is similar to a block variant suggested for the classical Davidson method applied to standard eigenproblems [26].

For simplicity assume that the matrix $\mathbf{B}$ is Hermitian positive definite. We construct a B-orthonormal basis with the modified Gram-Schmidt B-orthogonalization process $\left(\operatorname{Mod} G S_{B}\right)$, and solve the augmented correction equations (approximately) with $\mathbf{w}=\mathbf{u}$ and $\widetilde{\mathbf{w}}=\widetilde{\mathbf{u}}=\mathbf{B u}$. The block variant has the form as in Algorithm 9.3. Note that we try to obtain approximations for $\ell$ eigenvalues simultaneously. At every outer iteration we increase the dimension of the subspace $\mathcal{V}_{k}$ by $\ell$. The maximum dimension of the subspace is $m$. The restart is carried out with the $\ell$ current eigenvector approximations. 


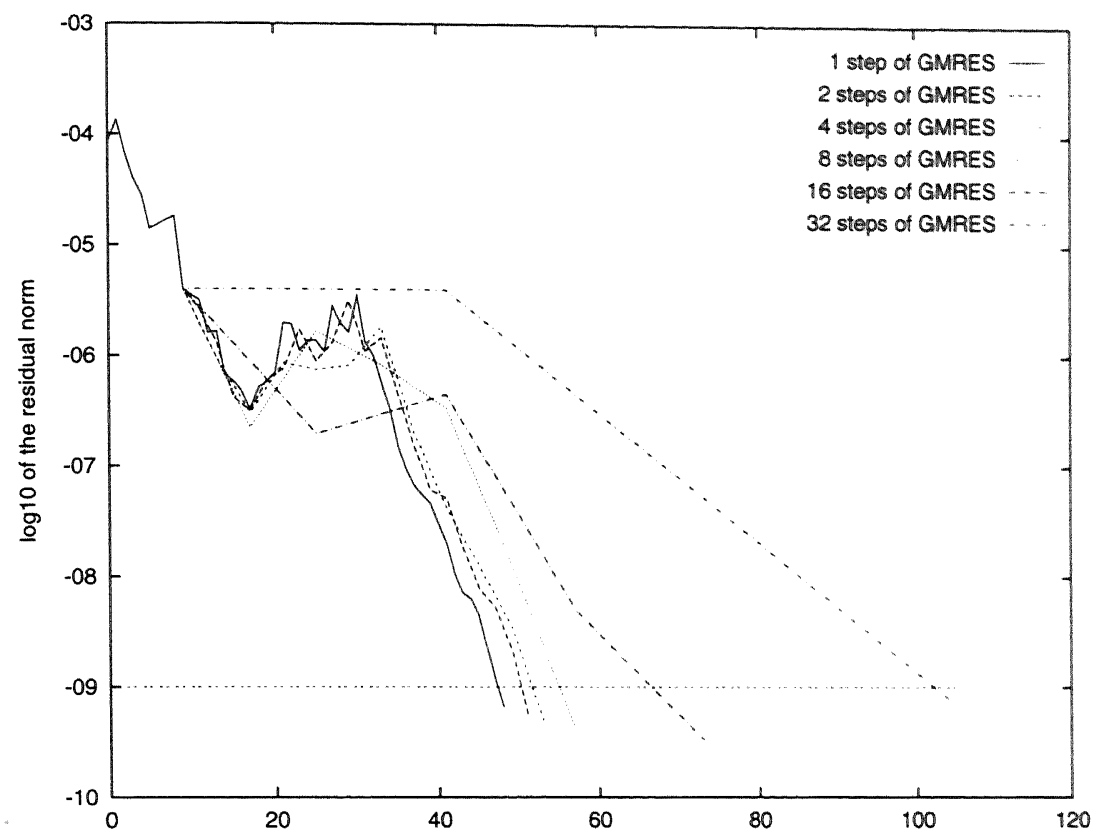

Figure 9.9: Example $\S 9.4$. Number of MVs.

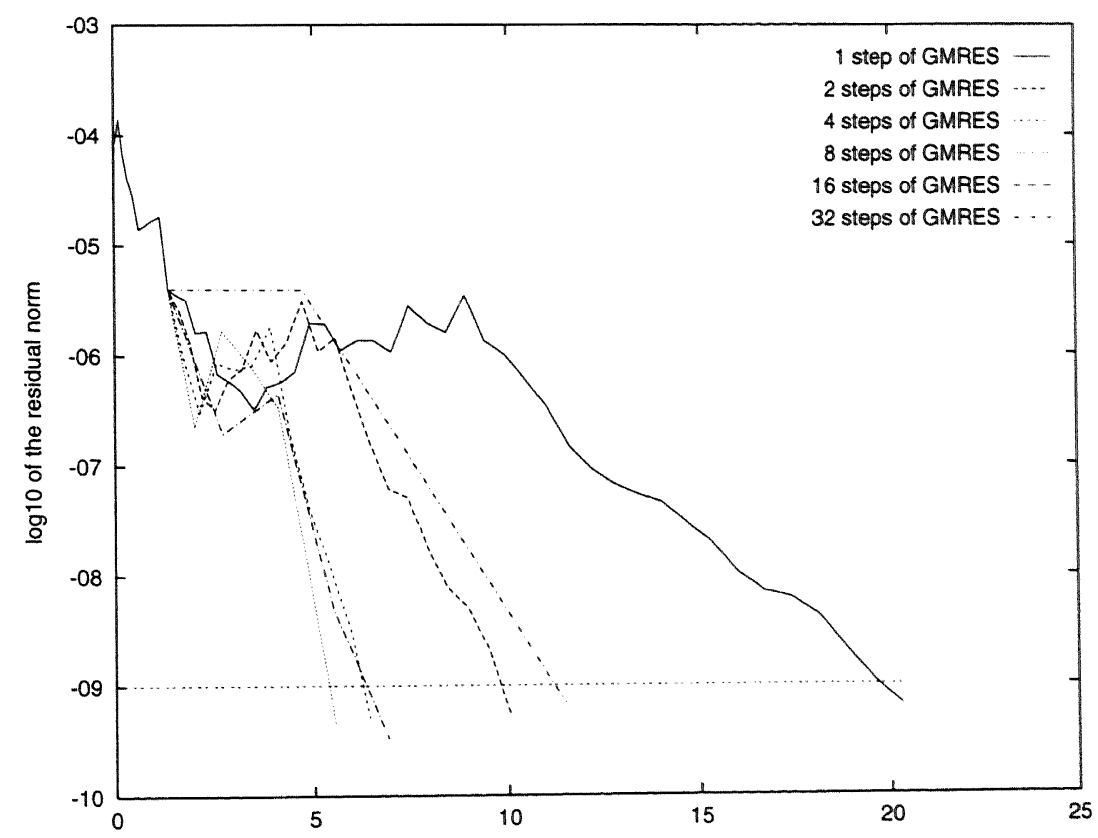

Figure 9.10: Example $§ 9.4$. Number of flops $\left(\times 10^{6}\right)$. 
Algorithm 9.3: A block variant of Jacobi-Davidson.

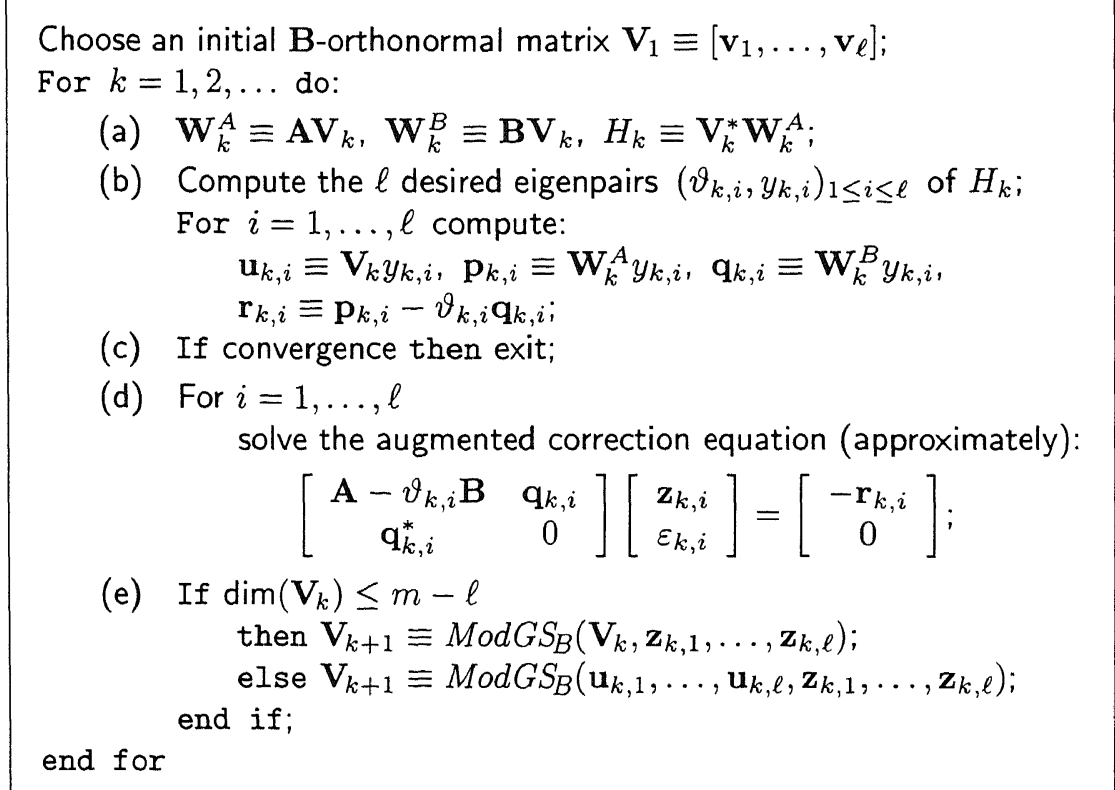

As an alternative block variant, one can also combine Algorithm 4.2 with the block variant discussed here, i.e., at a certain stage in Algorithm 4.2 one restarts and switches over to the block variant described above. Another possibility for computing several eigenvalues is to incorporate deflation techniques, see for instance $[24,30,9,23]$.

We have applied this block variant for the MHD test problem ( $\$ 9.3)$ and we have tried to reproduce the Alfvén spectrum. Therefore we have run the algorithm, for several suitably chosen targets, with $m=48$ and we have computed approximations for the $\ell=4$ eigenvalues nearest to the target. For the initial matrix we have chosen: $\mathbf{V}_{1}=\operatorname{Mod} G S_{B}\left(e_{201}, \ldots, e_{204}\right)$, with $e_{j}$ the $j$-th canonical unit vector. The eigenvector approximations $\mathbf{u}_{k, i}$ are normalized to have B-norm unity. The algorithm is stopped when the residual norm of the eigenpair approximation nearest to the target is smaller than $10^{-10}$.

We have solved the augmented correction equation with (at most) 100 steps of GMRES, using the same block Jacobi (left) preconditioning as in $§ 9.3$.

The results are given in Table 9.4. We have listed the target $(\tau)$, the number of outer iterations (\# iter), the number of converged eigenvalues (\# EVs), the minimum of the residual norms of the converged eigenpairs (min. res.), and the maximum of the residual norms of the converged eigenpairs (max. res.). We have used as criterion that an eigenpair approximation is considered to be converged when the residual norm is smaller than $10^{-6}$. For eigenvalues close to the 'bifurcation' point (see Fig. 9.5; targets 5 to 11) the results are obtained with $\ell=3$, 
Table 9.4: Example §9.5. Results of the block variant applied to the MHD test problem.

\begin{tabular}{|l|c|c|c|c|}
\hline target & \# iter & \# EVs & min. res. & max. res. \\
\hline$\tau_{1}=-0.6+0.25 \mathrm{i}$ & 16 & 3 & $6.06 \mathrm{E}-13$ & $1.62 \mathrm{E}-08$ \\
$\tau_{2}=-0.5+0.45 \mathrm{i}$ & 27 & 4 & $5.35 \mathrm{E}-13$ & $2.51 \mathrm{E}-07$ \\
$\tau_{3}=-0.325+0.525 \mathrm{i}$ & 22 & 4 & $9.94 \mathrm{E}-11$ & $8.83 \mathrm{E}-10$ \\
$\tau_{4}=-0.25+0.55 \mathrm{i}$ & 18 & 3 & $5.03 \mathrm{E}-12$ & $3.33 \mathrm{E}-09$ \\
$\tau_{5}=-0.2+0.65 \mathrm{i}$ & 26 & 3 & $1.42 \mathrm{E}-08$ & $3.06 \mathrm{E}-07$ \\
$\tau_{6}=-0.125+0.625 \mathrm{i}$ & 23 & 3 & $6.05 \mathrm{E}-08$ & $8.07 \mathrm{E}-07$ \\
$\tau_{7}=-0.075+0.625 \mathrm{i}$ & 26 & 3 & $5.03 \mathrm{E}-08$ & $5.06 \mathrm{E}-07$ \\
$\tau_{8}=-0.075+0.59 \mathrm{i}$ & 28 & 2 & $4.10 \mathrm{E}-08$ & $1.50 \mathrm{E}-07$ \\
$\tau_{9}=0.0+0.525 \mathrm{i}$ & 24 & 3 & $1.62 \mathrm{E}-08$ & $7.56 \mathrm{E}-08$ \\
$\tau_{10}=-0.04+0.68 \mathrm{i}$ & 27 & 3 & $2.35 \mathrm{E}-10$ & $1.45 \mathrm{E}-08$ \\
$\tau_{11}=-0.0275+0.6225 \mathrm{i}$ & 25 & 3 & $2.58 \mathrm{E}-08$ & $4.48 \mathrm{E}-07$ \\
\hline
\end{tabular}

$m=45$ and stopping tolerance $10^{-7}$, starting with $\mathbf{V}_{1}=\operatorname{Mod} G S_{B}\left(e_{201}, \ldots, e_{203}\right)$. The motivation for this is that in that region the eigenvalues are very close and consequently the eigenpairs are difficult to distinguish. This has also been experienced in ref. [16] for the generalized Lanczos method as well as for the shiftand-invert Arnoldi method. From Table 9.4 we see that 11 targets have been necessary to reproduce the entire Alfvén spectrum (34 eigenvalues for this test problem).

\section{Acknowledgement.}

We gratefully acknowledge stimulating discussions with Martin van Gijzen.

\section{REFERENCES}

1. E. Anderson, Z. Bai, C. Bischof, J. Demmel, J. Dongarra, J. D. Croz, A. Greenbaum, S. Hammarling, A. McKenney, S. Ostrouchov, and D. Sorensen, LAPACK Users' Guide, SIAM, Philadelphia, 1992.

2. Z. Bai, Progress in the numerical solution of the nonsymmetric eigenvalue problem, Numer. Linear Algebra Appl., 2 (1995), pp. 219-234.

3. Z. Bai, R. Barret, D. Day, J. Demmel, and J. Dongarra, Test matrix collection (non-Hermitian eigenvalue problems), Tech. Report, June 61995.

4. J. G. L. Booten, H. A. Van der Vorst, P. M. Meijer, and H. J. J. te Riele, A preconditioned Jacobi-Davidson method for solving large generalized eigenvalue problems, Report NM-R9414, Dept. Num. Math., CWI, Amsterdam, 1994.

5. M. Crouzeix, B. Philippe, and M. Sadkane, The Davidson method, SIAM J. Sci. Comput., 15 (1994), pp. 62-76.

6. J. Cullum, W. Kerner, and R. Willoughby, A generalized nonsymmetric Lanczos procedure, Comput. Phys. Commun., 53 (1989), pp. 19-48.

7. E. R. Davidson, The iterative calculation of a few of the lowest eigenvalues and corresponding eigenvectors of large real symmetric matrices, J. Comp. Phys., 17 (1975), pp. 87-94. 
8. E. R. Davidson, Monster matrices: Their eigenvalues and eigenvectors, Computers in Physics, (1993), pp. 519-522.

9. D. R. Fokkema, G. L. G. Sleijpen, and H. A. Van der Vorst, Accelerated inexact Newton schemes for large systems of nonlinear equations, Preprint 918, Dep. Math., Utrecht University, July 1995.

10. D. R. Fokkema, G. L. G. Sleijpen, and H. A. Van der Vorst, Jacobi-Davidson style $Q R$ and $Q Z$ algorithms for the reduction of matrix pencils, Preprint 941, Dep. Math., University Utrecht, January 1996. To appear in SIAM J. Sci. Comput.

11. R. Glowinski, H. B. Keller, and L. Reinhart, Continuation-conjugate gradient methods for the least squares solution of nonlinear boundary value problems, SIAM J. Sci. Statist. Comput., 6 (1985), pp. 793-832.

12. J. P. Goedbloed, S. Poedts, G. T. A. Huysmans, G. Halberstadt, H. Holties, and A. J. C. Beliën, Magnetohydrodynamic spectroscopy: Large scale computation of the spectrum of waves in plasmas, Future Generation Computer Systems, 10 (1994), pp. 339-343.

13. G. H. Golub and C. F. van Loan, Matrix Computations, 2nd ed., The Johns Hopkins University Press, Baltimore, MD, 1989.

14. C. G. J. Jacobi, Ueber eine neue Auflösungsart der bei der Methode der kleinsten Quadrate vorkommende linearen Gleichungen, Astronomische Nachrichten, (1845), pp. 297-306.

15. C. G. J. Jacobi, Ueber ein leichtes Verfahren, die in der Theorie der Säcularstörungen vorkommenden Gleichungen numerisch aufzulösen, Journal für die reine und angewandte Mathematik (Crelle's J.), 30 (1846), pp. 51-94. Reprinted in the collected works of Jacobi, Opuscula Mathematica.

16. M. N. Kooper, H. A. Van der Vorst, S. Poedts, and J. P. Goedbloed, Application of the implicitly updated Arnoldi method with a complex shift and invert strategy in MHD, J. Comput. Phys., 118 (1995), pp. 320-328.

17. R. B. Morgan, Computing interior eigenvalues of large matrices, Linear Algebra Appl., 154/156 (1991), pp. 289-309.

18. R. B. Morgan, Generalizations of Davidson's method for computing eigenvalues of large nonsymmetric matrices, J. Comput. Phys., 101 (1992), pp. 287-291.

19. R. B. Morgan and D. S. Scott, Generalizations of Davidson's method for computing eigenvalues of sparse symmetric matrices, SIAM J. Sci. Comput., 7 (1986), pp. 817 825.

20. R. B. Morgan and D. S. Scott, Preconditioning the Lanczos algorithm for sparse symmetric eigenvalue problems, SIAM J. Sci. Comput., 14 (1993), pp. 585-593.

21. J. Olsen, P. Jørgensen, and J. Simons, Passing the one-billion limit in full configuration-interaction (FCI) calculations, Chemical Physics Letters, 169 (1990), pp. $463-472$.

22. C. C. Paige, B. N. Parlett, and H. A. Van der Vorst, Approximate solutions and eigenvalue bounds from Krylov subspaces, Numer. Linear Algebra Appl., 2 (1995), pp. 115-134.

23. F. A. Raeven and M. B. van Gijzen, The parallel computation of the smallest eigenpair of an acoustic problem with damping. Preprint 924, Dep. Math., University Utrecht, September, 1995.

24. Y. Saad, Numerical Methods for Large Eigenvalue Problems, Manchester University Press, Manchester, U.K., 1992. 
25. Y. Saad and M. H. Schultz, GMRES: A generalized minimum residual algorithm for solving nonsymmetric linear systems, SIAM J. Sci. Statist. Comput., 7 (1986), pp. 856-869.

26. M. Sadkane, Block-Arnoldi and Davidson methods for unsymmetric large eigenvalue problems, Numer. Math., 64 (1993), pp. 195-211.

27. A. H. Sameh and J. A. Wisniewski, A trace minimization algorithm for the generalized eigenvalue problem, SIAM J. Numer. Anal., 19 (1982), pp. 1243-1259.

28. D. S. Scott, Solving sparse symmetric generalized eigenvalue problems without factorization, SIAM J. Numer. Anal., 18 (1981), pp. 102-110.

29. G. L. G. Sleijpen and H. A. Van der Vorst, A Jacobi-Davidson iteration method for linear eigenvalue problems, SIAM J. Matrix Anal. Appl., 17 (1996), pp. 401-425.

30. G. L. G. Sleijpen and H. A. Van der Vorst, The Jacobi-Davidson method for eigenvalue problems and its relation to accelerated inexact newton schemes, in Iterative Methods in Linear Algebra, II, Proceedings of the "Second IMACS International Symposium on Iterative Methods in Linear Algebra", Blagoevgrad, 1995, S. D. Margenov and P. S. Vassilevski, eds, Vol. 3 in the IMACS Series in Computational and Applied Mathematics, 1996.

31. D. C. Sorensen, Implicit application of polynomial filters in a k-step Arnoldi method, SIAM J. Matrix Anal. Appl., 13 (1992), pp. 357-385.

32. H. J. J. van Dam, J. H. van Lenthe, G. L. G. Sleijpen, and H. A. Van der Vorst, An improvement of Davidson's iteration method; applications to MRCI and MRCEPA calculations. J. Comput. Chem., 17 (1996), pp. 267-272. 